\title{
WEYL LAW FOR THE VOLUME SPECTRUM
}

\author{
YEVGENY LIOKUMOVICH, FERNANDO C. MARQUES, AND ANDRÉ NEVES
}

\begin{abstract}
Given $M$ a Riemannian manifold with (possibly empty) boundary, we show that its volume spectrum $\left\{\omega_{p}(M)\right\}_{p \in \mathbb{N}}$ satisfies a Weyl law that was conjectured by Gromov.
\end{abstract}

\section{INTRODUCTION}

Let $(M, g)$ be a compact Riemannian manifold of dimension $n+1$. It is well known that the eigenvalues of the Laplacian have the following min-max characterization:

$$
\lambda_{p}=\inf _{p-\text { plane } Q \subset \mathrm{W}^{1,2}(\mathrm{M})} \sup _{f \in Q-\{0\}} \frac{\int_{M}|\nabla f|^{2} d V}{\int_{M} f^{2} d V}, \quad p \in \mathbb{N} .
$$

In 1911, Weyl ([22]) proved an asymptotic formula for the sequence of eigenvalues $\left\{\lambda_{p}\right\}_{p \in \mathbb{N}}$ that had a tremendous impact in Mathematics. The celebrated Weyl law states that

$$
\lim _{p \rightarrow \infty} \lambda_{p} p^{-\frac{2}{n+1}}=a(n) \operatorname{vol}(M)^{-\frac{2}{n+1}},
$$

where $a(n)=4 \pi^{2} \operatorname{vol}(B)^{-\frac{2}{n+1}}$ and $B$ is the unit ball in $\mathbb{R}^{n+1}$.

Gromov ([5], [6, Section 8], [7, Section 5.2], [8]) proposed a very general framework to study several non-linear analogs of the spectral problem on $M$. In the case we are interested, the space $W^{1,2}(M)$ is replaced by the space $\mathcal{Z}_{n}\left(M ; \mathbb{Z}_{2}\right)$ of $\bmod 2$ flat $n$-cycles in $M$ (if $M$ has no boundary) and the energy functional is replaced by the volume functional (see Section 2.1 for precise definitions). Almgren [1] showed there is a weak homotopy equivalence between $\mathcal{Z}_{n}\left(M ; \mathbb{Z}_{2}\right)$ and $\mathbb{R P} \mathbb{P}^{\infty}$ and thus its cohomology ring has a generator $\bar{\lambda} \in H^{1}\left(\mathcal{Z}_{n}\left(M ; \mathbb{Z}_{2}\right) ; \mathbb{Z}_{2}\right)$. Instead of considering $p$-planes in $W^{1,2}(M)$ one considers $p$-sweepouts, i.e., subsets of $\mathcal{Z}_{n}\left(M ; \mathbb{Z}_{2}\right)$ where the $p$-th cup power $\bar{\lambda}^{p}$ does not vanish (see Section 2.5 for precise definitions). The definition of width is similar to the above min-max characterization of the eigenvalues. The $p$-width of $M$, denoted by $\omega_{p}(M)$, is defined as the infimum over all real numbers $w$, such that there exists a $p$-sweepout with every element having volume at most $w$ (see Section 2.6 for precise definitions or [9] for some

The article was partly written during the first author's visit to Max Planck Institute for Mathematics at Bonn; he is grateful to the Institute for its hospitality. The second author was partly supported by NSF-DMS-1509027 and NSF DMS-1311795. The third author was partly supported by ERC-2011-StG-278940 and EPSRC Programme Grant $\mathrm{EP} / \mathrm{K} 00865 \mathrm{X} / 1$. 
motivation). In the same way that eigenvalues are realized by the energy of eigenfunctions, Almgren-Pitts Min-max Theory says that the widths are realized by the volume of minimal surfaces (with a possibly small singular set). Similar considerations apply when $M$ has boundary but one has to use the space $\mathcal{Z}_{n, \text { rel }}\left(M, \partial M ; \mathbb{Z}_{2}\right)$ of relative $\bmod 2$ flat cycles.

An insightful idea of Gromov was to understand that, using the cohomology structure of $\mathcal{Z}_{n}\left(M ; \mathbb{Z}_{2}\right)$, many properties of the energy spectrum $\left\{\lambda_{p}\right\}_{p \in \mathbb{N}}$ can be extended to the volume spectrum $\left\{\omega_{p}(M)\right\}_{p \in \mathbb{N}}$. For instance, Gromov and later Guth ([6] or Guth [9]) showed the existence of a constant $C=C(M, g)$ for which

$$
C^{-1} \operatorname{vol}(M)^{\frac{n}{n+1}} p^{\frac{1}{n+1}} \leq \omega_{p}(B) \leq C \operatorname{vol}(M)^{\frac{n}{n+1}} p^{\frac{1}{n+1}}
$$

for all $p \in \mathbb{N}$.

The asymptotic behaviour of the volume spectrum has been studied in the paper by Guth $([9])$. It has been used by Marques and Neves to prove existence of infinitely many minimal hypersurfaces in manifolds with positive Ricci curvature [16].

Gromov conjectured $([6,8.4])$ that the volume spectrum $\left\{\omega_{p}(M)\right\}_{p \in \mathbb{N}}$ satisfies a Weyl's asymptotic law. In this paper we confirm this and show

1.1. Weyl Law for the Volume Spectrum. There exists a constant $a(n)>0$ such that, for every compact Riemannian manifold $\left(M^{n+1}, g\right)$ with (possibly empty) boundary, we have

$$
\lim _{p \rightarrow \infty} \omega_{p}(M) p^{-\frac{1}{n+1}}=a(n) \operatorname{vol}(M)^{\frac{n}{n+1}} .
$$

After our paper was completed, the Weyl Law for the Volume Spectrum was used in a fundamental way in [12] to prove the following theorem:

Theorem (Irie, Marques, Neves, 2017): Let $M^{n+1}$ be a closed manifold of dimension $(n+1)$, with $3 \leq(n+1) \leq 7$. Then for a $C^{\infty}$-generic Riemannian metric $g$ on $M$, the union of all closed, smooth, embedded minimal hypersurfaces is dense.

This theorem settles the generic case of Yau's Conjecture ([23]) about the existence of infinitely many minimal surfaces by proving that a much stronger property holds true: there are infinitely many closed embedded minimal hypersurfaces intersecting any given ball in $M$.

1.2. Let $\Omega$ be a bounded open subset of $\mathbb{R}^{n+1}$ with smooth boundary, or more generally, a Lipschitz domain in the sense of [11, Definition 2.5] (this is a weaker regularity condition for the boundary of $\Omega$, see Section 2.1). For such domains we have a more general Weyl law, which applies to the space of cycles of dimension $0<k \leq n$. Assume in addition that

$$
H_{i}\left(\Omega, \partial \Omega ; \mathbb{Z}_{2}\right) \text { is } \mathbb{Z}_{2} \text { if } i=n+1 \text { and } 0 \text { if } k<i<n+1 \text {. }
$$


Similarly to the case of codimension 1 we can define the $p$-width of dimension $k, \omega_{p}^{k}(\Omega)$, to be the min-max quantity corresponding to the $p$-th cup power of the generator $\bar{\lambda} \in H^{n+1-k}\left(\mathcal{Z}_{k, \text { rel }}\left(\Omega, \partial \Omega ; \mathbb{Z}_{2}\right) ; \mathbb{Z}_{2}\right)$.

1.3. Weyl Law for Euclidean domains. There exists a constant a $(n, k)$ such that, for every Lipschitz domain $\Omega$ satisfying (1), we have

$$
\lim _{p \rightarrow \infty} \omega_{p}^{k}(\Omega) p^{-\frac{n+1-k}{n+1}}=a(n, k) \operatorname{vol}(\Omega)^{\frac{k}{n+1}} .
$$

In this setting the inequalities proven by Gromov and Guth for the widths $[6,9]$ become, for all $0<k<n$ and $p \in \mathbb{N}$,

$$
C(\Omega)^{-1} \operatorname{vol}(\Omega)^{\frac{k}{n+1}} p^{\frac{n+1-k}{n+1}} \leq \omega_{p}^{k}(\Omega) \leq C(\Omega) \operatorname{vol}(\Omega)^{\frac{k}{n+1}} p^{\frac{n+1-k}{n+1}}
$$

for some constant $C(\Omega)>0$. For general Riemannian metrics on $\Omega$ and $k=n$ such inequalities do not hold with a constant that depends only on the dimension. There are examples of metrics $g_{i}$ on the unit 3-ball $B^{3}$ with $\operatorname{vol}\left(B^{3}, g_{i}\right)=1$ and $\omega_{1}^{2}\left(B^{3}, g_{i}\right) \rightarrow \infty[19]$. Upper bounds for the widths can be obtained if the constant $C$ is allowed to depend on the conformal class of the manifold (see [4] and [14]), similarly to the upper bounds obtained by Korevaar for the eigenvalues of the Laplacian [13].

1.4. Overview of proof. We start by describing Weyl law for Lipschitz domains $\Omega$. One of the main tools in the proofs is the Lusternick-Schnirelman inequality, which relates the widths of $\Omega$ to the widths of its subsets (see [6, 8.3], [9, Section 3]). The idea behind this inequality is the following. Let $U_{1}$ and $U_{2}$ be two disjoint domains in $\Omega$ and let $V_{i} \subset \mathcal{Z}_{k \text {,rel }}\left(\Omega, \partial \Omega ; \mathbb{Z}_{2}\right)$ denote the set of all cycles whose restriction to $U_{i}$ has mass strictly less than $\omega_{1}^{k}\left(U_{i}\right)$, $i=1,2$. A sweepout of $\Omega$ restricts to a sweepout of $U_{i}$ and therefore the generator $\bar{\lambda}$ vanishes on $V_{i}, i=1,2$. If $V_{1}$ and $V_{2}$ are open, it follows that the cup power $\bar{\lambda}^{2}$ vanishes on $V_{1} \cup V_{2}$. Thus every 2-sweepout must have an element that does not lie in $V_{1} \cup V_{2}$ and this means that $\omega_{2}^{k}(\Omega) \geq \omega_{1}^{k}\left(U_{1}\right)+\omega_{1}^{k}\left(U_{2}\right)$. Iterating this argument we obtain that for $N$ disjoint domains $\left\{U_{i}\right\}_{i=1}^{N}$ in $\Omega$ and any $p \geq \sum_{i=1}^{N} p_{i}$ we have

$$
\omega_{p}^{k}(\Omega) \geq \sum_{i=1}^{N} \omega_{p_{i}}^{k}\left(U_{i}\right) .
$$

We first sketch how to prove the existence of the limit for the standard Euclidean cube $C$ of volume 1. To do this we exploit the fact that $C$ admits self-similar tilings.

Let $\tilde{\omega}_{p}^{k}(C)=\omega_{p}^{k}(C) p^{-\frac{n+1-k}{n+1}}$. By the upper bound in (2) the sequence $\left\{\tilde{\omega}_{p}^{k}(C)\right\}_{p \in \mathbb{N}}$ is bounded. Choose $p_{l}$ and $q_{j}$ so that $\tilde{\omega}_{p_{l}}^{k}(C)$ and $\tilde{\omega}_{q_{j}}^{k}(C)$ are, respectively, very close to $\lim \sup _{p \rightarrow \infty} \tilde{\omega}_{p}(C)$ and $\liminf \operatorname{in}_{p \rightarrow \infty} \tilde{\omega}_{p}(C)$, and $q_{j}$ is 
much bigger than $p_{l}$. Consider a maximal packing of $C$ by squares $\left\{C_{i}^{*}\right\}_{i=1}^{N}$ of volume $p_{l} / q_{j}$. From (3) we have

$$
\omega_{q_{j}}^{k}(C) \geq \sum_{i=1}^{N} \omega_{p_{l}}^{k}\left(C_{i}^{*}\right)=N\left(\frac{p_{l}}{q_{j}}\right)^{\frac{k}{n+1}} \omega_{p_{l}}^{k}(C)
$$

and thus

$$
\tilde{\omega}_{q_{j}}^{k}(C) \geq N \frac{p_{l}}{q_{j}} \tilde{\omega}_{p_{l}}^{k}(C)
$$

The maximal packing condition implies that, as $q_{j} \rightarrow \infty$, we have $N \frac{p_{l}}{q_{j}} \rightarrow 1$ and so limsup and lim inf must coincide. Thus we can denote this limit by a constant $a(n, k)$.

To prove that sequences $\left\{\tilde{\omega}_{p}^{k}(\Omega)\right\}$ converge to $a(n, k)$ for unit volume domains $\Omega$ in $\mathbb{R}^{n+1}$ we use similar arguments but applied to efficient packings of scaled copies of $C$ in $\Omega$ and scaled copies of $\Omega$ in $C$.

For a Riemannian manifolds $(M, g)$ a crucial difficulty arises because while one can find efficient packings of cubes (or balls) in $M$, one cannot find efficient packings of copies of $M$ in a cube. The former implies that one can repeat the same type of arguments and show that, with $a(n)=a(n, n)$,

$$
\liminf _{p \rightarrow \infty} \omega_{p}(M) p^{-\frac{1}{n+1}} \geq a(n) \operatorname{vol}(M)^{\frac{n}{n+1}}
$$

but the latter implies that one needs another idea to prove the reverse inequality.

We do this by subdividing $M$ into $N$ small regions $\left\{Q_{i}\right\}_{i=1}^{N}$, which are $(1+\varepsilon)$-bilipschitz diffeomorphic to domains in $\mathbb{R}^{n+1}$. Mapping each $Q_{i}$ to $\mathbb{R}^{n+1}$ by the corresponding diffeomorphism and connecting the images of $Q_{i}$ by tubes of small total volume we obtain a connected domain $\Omega \subset \mathbb{R}^{n+1}$. A $p$ sweepout of $\Omega$ induces, via restriction, $p$-sweepouts of $Q_{i}, i=1, \ldots, N$. The elements in these restricted sweepouts have boundary in $\partial Q_{i}, i=1, \ldots, N$ and so we cannot add them to make a sweepout of $M$. However, we show that we can turn each restricted $p$-sweepout into a continuous family of cycles in $Q_{i}$ if we add to each element some chain that is entirely contained in $\partial Q_{i}, i=1, \ldots, N$. Combining these $N$ families of cycles we show that we obtain a $p$-sweepout of $M$ where the mass of each element, when compared with the corresponding element in $\mathcal{Z}_{n, \text { rel }}\left(\Omega, \partial \Omega ; \mathbb{Z}_{2}\right)$, has increased at most by the volume of $\cup_{i=1}^{N} \partial Q_{i}$. As $p \rightarrow \infty$ the volume of $\cup_{i=1}^{N} \partial Q_{i}$ is negligible compared to $p^{1 /(n+1)}$ yielding the desired upper bound. This is the only part in the argument where we restrict to the codimension 1 case.

We now mention two technical issues that arise in the proof of (3). The first issue is that the restriction of a cycle in $\mathcal{Z}_{k, \text { rel }}\left(\Omega, \partial \Omega ; \mathbb{Z}_{2}\right)$ to $U_{i}$ may not belong to $\mathcal{Z}_{k, \text { rel }}\left(U_{i}, \partial U_{i} ; \mathbb{Z}_{2}\right)$ because its boundary might have infinite mass and so we can not conclude that a sweepout of $\Omega$ restricts to a sweepout of $U_{i}$. To resolve this we use a perturbation argument and slicing theorem from geometric measure theory (see Lemma 2.15). The second issue is that mass is not a continuous function on the space of flat cycles and therefore the 
subsets $V_{1}$ and $V_{2}$ need not be open. To resolve this we follow the strategy in [15] and consider the finer topology of the mass norm on the space of relative cycles. We show that restricting to this finer topology does not increase the value of the width (see Section 2.9) and thus there is no loss of generality.

1.5. Some questions. We list some open questions.

The first question is to compute the constants $a(n, k)$. This is unknown even in the simplest case $n=k=1$. Potential candidates for the asymptotically optimal families of sweepouts include nodal sets of eigenfunctions on the flat disc or the round sphere, or zero sets of harmonic polynomials on the flat disc.

The second question is whether the argument for widths of Riemannian manifolds can be extended to higher codimension. Namely, is it true that for a compact Riemannian manifold

$$
\lim _{p \rightarrow \infty} \omega_{p}^{k}(M) p^{-\frac{n+1-k}{n+1}}=a(n, k) \operatorname{vol}(M)^{\frac{k}{n+1}}
$$

for $k<n$, where $a(n, k)$ is the constant for the corresponding limit for Euclidean domains? That the liminf of the sequence on the left side is greater or equal than the right side is shown in Theorem 4.1.

In the case of higher codimension, the cohomology ring of the space of relative cycles is richer (see [9]) and so another question would be to understand the asymptotic limit for the widths associated with Steenrod powers.

The paper is organized as follows. In Section 2 we give necessary definitions and prove some technical results that we need for the proof of Lusternick-Schnirelman inequality. In Section 3 we prove Lusternick-Schnirelman inequality and Theorem 1.3. In Section 4 we prove Theorem 1.1.

\section{Definitions And SETUP}

2.1. Geometric Measure Theory. Given $m \in \mathbb{N}, I^{m}$ denotes the $m$ dimensional cube $I^{m}=[0,1]^{m}$. For each $j \in \mathbb{N}, I(1, j)$ denotes the cube complex on $I^{1}$ whose 1 -cells and 0 -cells (those are sometimes called vertices) are, respectively,

$$
\left[0,3^{-j}\right],\left[3^{-j}, 2 \cdot 3^{-j}\right], \ldots,\left[1-3^{-j}, 1\right] \text { and }[0],\left[3^{-j}\right], \ldots,\left[1-3^{-j}\right],[1] .
$$

We denote by $I(m, j)$ the cell complex on $I^{m}$ :

$$
I(m, j)=I(1, j) \otimes \ldots \otimes I(1, j) \quad(m \text { times }) .
$$

Then $\alpha=\alpha_{1} \otimes \cdots \otimes \alpha_{m}$ is a $q$-cell of $I(m, j)$ if and only if $\alpha_{i}$ is a cell of $I(1, j)$ for each $i$, and $\sum_{i=1}^{m} \operatorname{dim}\left(\alpha_{i}\right)=q$. We often abuse notation by identifying a $q$-cell $\alpha$ with its support: $\alpha_{1} \times \cdots \times \alpha_{m} \subset I^{m}$.

Given $X$ a cubical subcomplex of $I^{m}$, the cube complex $X(j)$ is the union of all cells of $I(m, j)$ whose support is contained in some cell of $X$. We use the notation $X(j)_{q}$ to denote the set of all $q$-cells in $X(j)$. 
The ambient spaces considered in this paper are compact Riemannian $(n+1)$-manifolds $\left(M^{n+1}, g\right)$ with smooth and possibly empty boundary $\partial M$. We can always assume $M$ is isometrically embedded in some Euclidean space $\mathbb{R}^{Q}$. We denote by $B_{r}(p)$ the Euclidean open ball of radius $r$ centered at $p \in \mathbb{R}^{Q}$.

When $M$ is a region of $\mathbb{R}^{n+1}$ we allow for less regularity and require $M$ to be a compact region with finite perimeter with the extra property that for all $p \in \partial M$ there is a hyperplane $H \subset \mathbb{R}^{n+1}$ containing $p, r>0$, and a Lipschitz function $\phi$ defined on $H \cap B_{r}(p)$ such that, denoting by $N$ a normal vector to $H$,

$$
M \cap B_{r}(p)=B_{r}(p) \cap\{x+t N: x \in H, t \geq \phi(x)\} .
$$

We call these regions Lipschitz domains (see [11, Definition 2.5]).

For reasons to be explained in Section 2.5, with $0 \leq k<n+1$ fixed, we also assume that

$$
H_{i}\left(M, \partial M ; \mathbb{Z}_{2}\right) \text { is } \mathbb{Z}_{2} \text { if } i=n+1 \text { and } 0 \text { if } k<i<n+1 .
$$

When $k=n$, this amounts to require that $M$ is connected.

The following definitions can be found in [2, Section 4.1]. For every $0 \leq$ $k \leq n+1, \mathcal{R}_{k}\left(M ; \mathbb{Z}_{2}\right)$ (or $\mathcal{R}_{k}\left(\partial M ; \mathbb{Z}_{2}\right)$ ) denotes the set of $k$-dimensional rectifiable mod 2 flat chains in $\mathbb{R}^{Q}$ whose support lies in $M$ (or $\partial M$ ). The Radon measure in $M$ associated with $T \in \mathcal{R}_{k}\left(M ; \mathbb{Z}_{2}\right)\left(\right.$ or $\left.\mathcal{R}_{k}\left(\partial M ; \mathbb{Z}_{2}\right)\right)$ is denoted by $\|T\|$, and its support is denoted by $\operatorname{supp}(T)$.

The mass $\mathbf{M}$ of $T \in \mathcal{R}_{k}\left(M ; \mathbb{Z}_{2}\right)$ is defined in [2, p. 358]. With $S, T \in$ $\mathcal{R}_{k}\left(M ; \mathbb{Z}_{2}\right)$ the flat metric is given by

$$
\begin{aligned}
\mathcal{F}(T, S)=\inf \{\mathbf{M}(Q)+\mathbf{M}(R): \quad & T-S=R+\partial Q, \\
& \left.R \in \mathcal{R}_{k}\left(M ; \mathbb{Z}_{2}\right), Q \in \mathcal{R}_{k+1}\left(M ; \mathbb{Z}_{2}\right)\right\} .
\end{aligned}
$$

Given a Lipschitz map $F: M \rightarrow M$, the push-forward of $T \in \mathcal{R}_{k}\left(M ; \mathbb{Z}_{2}\right)$ is denoted by $F_{\#}(T)$.

If $k \geq 1, \mathbf{I}_{k}\left(M ; \mathbb{Z}_{2}\right)$ (or $\left.\mathbf{I}_{k}\left(\partial M ; \mathbb{Z}_{2}\right)\right)$ denotes those elements of $\mathcal{R}_{k}\left(M ; \mathbb{Z}_{2}\right)$ whose boundary lies in $\mathcal{R}_{k-1}\left(M ; \mathbb{Z}_{2}\right)$. Finally, we also consider the spaces

$$
\mathcal{Z}_{k}\left(M ; \mathbb{Z}_{2}\right)=\left\{T \in \mathbf{I}_{k}\left(M ; \mathbb{Z}_{2}\right): \partial T=0\right\}
$$

and

$$
\mathcal{Z}_{k}\left(M, \partial M ; \mathbb{Z}_{2}\right)=\left\{T \in \mathbf{I}_{k}\left(M ; \mathbb{Z}_{2}\right): \operatorname{support}(\partial T) \subset \partial M\right\} .
$$

2.2. Relative cycles. We now describe the space of relative cycles following $\left[1\right.$, Definition 1.20]. We say that $T, S \in \mathcal{Z}_{k}\left(M, \partial M ; \mathbb{Z}_{2}\right)$ are equivalent if $T-S \in \mathbf{I}_{k}\left(\partial M ; \mathbb{Z}_{2}\right)$ and the space of such equivalence classes is denoted by $\mathcal{Z}_{k, \text { rel }}\left(M, \partial M ; \mathbb{Z}_{2}\right)$. There is a natural projection map

$$
P: \mathcal{Z}_{k}\left(M, \partial M ; \mathbb{Z}_{2}\right) \rightarrow \mathcal{Z}_{k, \text { rel }}\left(M, \partial M ; \mathbb{Z}_{2}\right) .
$$

If $U \subset \mathbb{R}^{Q}$ is an open set and $P(T)=P(S)$ then $T\llcorner(U \backslash \partial M)=S\llcorner(U \backslash \partial M)$.

When $\partial M=\emptyset$ then $\mathcal{Z}_{k \text {,rel }}\left(M, \partial M ; \mathbb{Z}_{2}\right)$ is identical to $\mathcal{Z}_{k}\left(M ; \mathbb{Z}_{2}\right)$. 
The flat metric and the mass norm in the space of relative cycles are defined, respectively, as

$$
\mathcal{F}(P(S), P(T))=\inf \left\{\mathcal{F}(S+R, T): R \in \mathbf{I}_{k}\left(\partial M ; \mathbb{Z}_{2}\right)\right\}
$$

or

$$
\mathbf{M}(P(T))=\inf \left\{\mathbf{M}(T+R): R \in \mathbf{I}_{k}\left(\partial M ; \mathbb{Z}_{2}\right)\right\} .
$$

These definitions do not depend on the choice of $S, T \in \mathcal{Z}_{k}\left(M, \partial M ; \mathbb{Z}_{2}\right)$. The flat topology on either $\mathcal{Z}_{k}\left(M, \partial M ; \mathbb{Z}_{2}\right)$ or $\mathcal{Z}_{k, \text { rel }}\left(M, \partial M ; \mathbb{Z}_{2}\right)$ denotes the topology induced by the flat metric. With the topology of the mass norm, the spaces will be denoted by $\mathcal{Z}_{k}\left(M, \partial M ; \mathbf{M} ; \mathbb{Z}_{2}\right)$ or $\mathcal{Z}_{k, \text { rel }}\left(M, \partial M ; \mathbf{M} ; \mathbb{Z}_{2}\right)$.

To keep notation simple, we denote $P(T) \in \mathcal{Z}_{k, \text { rel }}\left(M, \partial M ; \mathbb{Z}_{2}\right)$ by $T$.

\subsection{Federer-Fleming Compactness Theorem. The set}

$$
\left\{T \in \mathcal{Z}_{k, \text { rel }}\left(M, \partial M ; \mathbb{Z}_{2}\right): \mathbf{M}(T) \leq L\right\}
$$

is compact in the flat topology for all $L>0$.

Proof. There is an open neighborhood $U \subset \mathbb{R}^{Q}$ of $\partial M$ and a Lipschitz map $F: U \longrightarrow M$ so that $F(x)=x$ for all $x \in \partial M$. If $\partial M$ is smooth this is obvious while if $M$ is a Lipschitz domain this follows from [11, (4.6.7)]. Set $\Lambda_{r}=\left\{x \in \mathbb{R}^{Q}: \operatorname{dist}(x, \partial M)<r\right\}$ and choose $r_{0}$ so that $\Lambda_{r_{0}} \subset U$.

It suffices to consider a sequence $\left\{P\left(T_{i}\right)\right\}_{i \in \mathbb{N}} \subset \mathcal{Z}_{k, \text { rel }}\left(M, \partial M ; \mathbb{Z}_{2}\right)$ such that the mass of $T_{i} \in \mathcal{Z}_{k}\left(M, \partial M ; \mathbb{Z}_{2}\right)$ is bounded by $2 L$. Slicing [21, Section 28] gives us, for all $i \in \mathbb{N}, 0<r_{i}<r_{0}$ so that

- $C_{i}=T_{i}\left\llcorner\Lambda_{r_{i}} \in \mathbf{I}_{k}\left(U ; \mathbb{Z}_{2}\right)\right.$;

- $\mathbf{M}\left(\partial C_{i}-\partial T_{i}\right) \leq 2 L / r_{0}$.

From the fact that $F_{\#}\left(\partial T_{i}\right)=\partial T_{i}$ we obtain that $\mathbf{M}\left(\partial F_{\#}\left(C_{i}\right)-\partial T_{i}\right)$ is uniformly bounded independently of $i \in \mathbb{N}$. Moreover $F_{\#}\left(C_{i}\right) \in \mathbf{I}_{k}(\partial M)$ for all $i \in \mathbb{N}$ and so $S_{i}=T_{i}-F_{\#}\left(C_{i}\right)$ is a sequence in $\mathcal{Z}_{k}\left(M, \partial M ; \mathbb{Z}_{2}\right)$ with bounded mass and bounded boundary mass. The result then follows from the classical Federer-Fleming Compactness Theorem.

The following proposition will also be needed.

2.4. Proposition. Given $T \in \mathcal{Z}_{k, \text { rel }}\left(M, \partial M ; \mathbb{Z}_{2}\right), \mathbf{M}(T)=\|T\|(M \backslash \partial M)$ and the mass is lower semicontinuous with respect to the flat topology in $\mathcal{Z}_{k, \text { rel }}\left(M, \partial M ; \mathbb{Z}_{2}\right)$ meaning that if $U \subset \mathbb{R}^{Q}$ is an open set and $\left\{T_{i}\right\}_{i \in \mathbb{N}}$ a sequence converging to $T$ in the flat topology then

$$
\|T\|(U \backslash \partial M) \leq \liminf _{i \rightarrow \infty}\left\|T_{i}\right\|(U \backslash \partial M) .
$$

In particular, $\mathbf{M}(T) \leq \liminf _{i \rightarrow \infty} \mathbf{M}\left(T_{i}\right)$.

Proof. Consider the projection map $F: U \longrightarrow M$ so that $F(x)=x$ for all $x \in \partial M$ and $U \subset \mathbb{R}^{Q}$ is an open neighborhood of $\partial M$.

Let $\Omega_{r}=\left\{x \in \mathbb{R}^{Q}: \operatorname{dist}(x, \partial M)>r\right\}$. The current $T\left\llcorner\Omega_{0}\right.$ is also rectifiable $[3,3.8(3)]$ and hence $S=T-T\left\llcorner\Omega_{0}\right.$ is a rectifiable current with support in $\partial M$. From the definition of rectifiable currents, this means there is a 
sequence of integral Lipschitz chains $S_{i} \in \mathbf{I}_{k}\left(\mathbb{R}^{Q}\right)$ with $\mathbf{M}\left(S-S_{i}\right) \rightarrow 0$ as $i \rightarrow \infty$. The currents $\left\{S_{i}\right\}_{i \in \mathbb{N}}$ can be chosen to have support in $U$ and thus, because $F_{\#}(S)=S$, we obtain that $F_{\#}\left(S_{i}\right) \in \mathbf{I}_{k}(\partial M)$ is such that $\mathbf{M}\left(S-F_{\#}\left(S_{i}\right)\right) \rightarrow 0$ as $i \rightarrow \infty$. Therefore $\mathbf{M}\left(T-F_{\#}\left(S_{i}\right)\right)$ tends to $\mathbf{M}\left(T\left\llcorner\Omega_{0}\right)\right.$ and so

$$
\mathbf{M}(P(T)) \leq \mathbf{M}\left(T\left\llcorner\Omega_{0}\right)=\|T\|(M \backslash \partial M) .\right.
$$

The opposite inequality is simple to check.

Without loss of generality we assume that the mass of $\left\{T_{i}\right\}_{i \in \mathbb{N}}$ is uniformly bounded in $\mathbf{I}_{k}\left(M ; \mathbb{Z}_{2}\right)$. From [1, Proposition 1.16] we can choose $\left\{r_{j}\right\}_{j \in \mathbb{N}}$ converging to zero such that, for all $j \in \mathbb{N}, T_{i}\left\llcorner\Omega_{r_{j}} \in \mathbf{I}_{k}\left(M ; \mathbb{Z}_{2}\right)\right.$ tends to $T\left\llcorner\Omega_{r_{j}} \in \mathbf{I}_{k}\left(M ; \mathbb{Z}_{2}\right)\right.$ in the flat topology as $i \rightarrow \infty$. Thus lower semicontinuity of the mass for integral currents implies that

$\|T\|\left(U \cap \Omega_{r_{j}}\right)=\| T\left\llcorner\Omega_{r_{j}}\left\|(U) \leq \liminf _{i \rightarrow \infty}\right\| T_{i}\left\llcorner\Omega_{r_{j}}\left\|(U) \leq \liminf _{i \rightarrow \infty}\right\| T_{i} \|(U \backslash \partial M)\right.\right.$.

Making $j \rightarrow \infty$ implies the desired result.

2.5. Almgren Isomorphism. We will be succinct and just describe the main concepts. The reader can see $[1,16,17,18]$ for some background and explicit constructions.

In [1] Almgren constructed, for each pair of integers $0 \leq k \leq n+1$ and $l \geq 1$, an isomorphism

$$
\Lambda_{l, \mathrm{rel}}: \pi_{l}\left(\mathcal{Z}_{k, \mathrm{rel}}\left(M, \partial M ; \mathbb{Z}_{2}\right),\{0\}\right) \rightarrow H_{k+l}\left(M, \partial M ; \mathbb{Z}_{2}\right) .
$$

When $\partial M=\emptyset, H_{k+l}\left(M, \partial M ; \mathbb{Z}_{2}\right)$ is identical to $H_{k+l}\left(M ; \mathbb{Z}_{2}\right)$.

The homological assumptions on $M$ (see Section 2.1) and the Almgren Isomorphism Theorem [1] imply that the homotopy groups of

$$
\left(\mathcal{Z}_{k, \text { rel }}\left(M, \partial M ; \mathbb{Z}_{2}\right),\{0\}\right)
$$

are all trivial except for the $(n+1-k)$-th one, i.e., $\mathcal{Z}_{k, \text { rel }}\left(M, \partial M ; \mathbb{Z}_{2}\right)$ is an Eilenberg-MacLane space $K\left(\mathbb{Z}_{2}, n+1-k\right)$. Thus from the Hurewicz Theorem and Universal Coefficients Theorem one has

$$
\begin{gathered}
H^{n+1-k}\left(\mathcal{Z}_{k, \text { rel }}\left(M, \partial M ; \mathbb{Z}_{2}\right) ; \mathbb{Z}_{2}\right)=\mathbb{Z}_{2}=\left\{0, \bar{\lambda}_{k}\right\} \\
H^{n+1-k}\left(\mathcal{Z}_{k, \text { rel }}\left(M, \partial M ; \mathbb{Z}_{2}\right) ; \mathbb{Z}_{2}\right)=\operatorname{Hom}\left(H_{n+1-k}\left(\mathcal{Z}_{k, \text { rel }}\left(M, \partial M ; \mathbb{Z}_{2}\right) ; \mathbb{Z}_{2}\right), \mathbb{Z}_{2}\right) .
\end{gathered}
$$

We now describe the map

$$
\bar{\lambda}_{k}: H_{n+1-k}\left(\mathcal{Z}_{k, \text { rel }}\left(M, \partial M ; \mathbb{Z}_{2}\right) ; \mathbb{Z}_{2}\right) \rightarrow \mathbb{Z}_{2} .
$$

An element $\sigma$ in $H_{n+1-k}\left(\mathcal{Z}_{k, \text { rel }}\left(M, \partial M ; \mathbb{Z}_{2}\right) ; \mathbb{Z}_{2}\right)$ is represented by a continuous map $\Phi: Y \rightarrow \mathcal{Z}_{k \text {,rel }}\left(M, \partial M ; \mathbb{Z}_{2}\right)$ where $Y$ is a $(n+1-k)$-cubical decomposition of the sphere with $\partial Y=0$.By Theorem 2.5 of [1], there exists a constant $\nu_{M, \partial M}>0$ such that for all $l \in \mathbb{N}$ with

$$
\mathcal{F}(\Phi(x), \Phi(y)) \leq \nu_{M, \partial M} \text { for all adjacent vertices } x, y \in Y(l)_{0},
$$

there exists a chain map $\phi: Y(l) \rightarrow \mathbf{I}_{*}\left(M ; \mathbb{Z}_{2}\right)$ of degree $k$ that extends $\Phi$ :

(i) $\phi$ coincides with $\Phi$ on $Y(l)_{0}$;

(ii) $\phi(\alpha) \in \mathbf{I}_{k+p}\left(M ; \mathbb{Z}_{2}\right)$ if $\alpha$ is a $p$-cell in $Y(l)_{p}$; 
(iii) $\partial \phi(\alpha)=\phi(\partial \alpha)$ in $\mathcal{Z}_{k+p \text {,rel }}\left(M, \partial M ; \mathbb{Z}_{2}\right)$ if $\alpha$ is a $(p+1)$-cell in $Y(l)_{p+1}$. Thus, for every $\alpha \in Y(l)_{n+1-k}$ we obtain $\phi(\alpha) \in \mathbf{I}_{n+1}\left(M ; \mathbb{Z}_{2}\right)$. From $\partial Y=0$ and (iii) we deduce that

$$
[\phi]:=\sum_{\alpha \in Y(l)_{n+1-k}} \phi(\alpha) \in \mathcal{Z}_{n+1, \operatorname{rel}}\left(M, \partial M ; \mathbb{Z}_{2}\right) .
$$

From the Constancy Theorem [21, Theorem 26.27], $[\phi]$ is either $M$ or 0 . In the first case $\bar{\lambda}_{k}(\sigma)=1$ and in the second case $\bar{\lambda}_{k}(\sigma)=0$.

2.6. Widths. Let $X$ denote a cubical subcomplex of the $m$-dimensional cube $I^{m}$. Given $p \in \mathbb{N}$ and an integer $0 \leq k<n+1$, a continuous map in the flat topology

$$
\Phi: X \rightarrow \mathcal{Z}_{k, \text { rel }}\left(M, \partial M ; \mathbb{Z}_{2}\right)
$$

is called a p-sweepout if the p-th cup power of $\lambda_{k}=\Phi^{*}\left(\bar{\lambda}_{k}\right)$ is nonzero in $H^{p(n+1-k)}\left(X ; \mathbb{Z}_{2}\right)$. The set of all $p$-sweepouts is closed under homotopies in the flat topology.

We denote by $\mathcal{P}_{p}^{k}(M)$ the set of all $p$-sweepouts that are continuous in the flat topology and have no concentration of mass, meaning that (see $[16$, Section 3.7]) setting

$$
\mathbf{m}(\Phi, r)=\sup \left\{\|\Phi(x)\|\left(B_{r}(p) \backslash \partial M\right): x \in \operatorname{dmn}(\Phi), p \in M\right\}
$$

we have $\lim _{r \rightarrow 0} \mathbf{m}(\Phi, r)=0$. The definition above is independent of the representative chosen for the relative cycle $\Phi(x)$.

Note that two maps in $\mathcal{P}_{p}^{k}(M)$ can have different domains.

2.7. Lemma. Continuous maps in the mass topology have no concentration of mass.

Proof. Consider $\bar{\Phi}: X \longrightarrow \mathcal{Z}_{k, \text { rel }}\left(M, \partial M ; \mathbb{Z}_{2}\right)$ a continuous map in the mass topology.

Choose $\delta>0$. From Proposition 2.4 we have that for all $x \in X$ there is $\Phi(x) \in \mathcal{Z}_{k}\left(M, \partial M ; \mathbb{Z}_{2}\right)$ with $P(\Phi(x))=\bar{\Phi}(x)$ and such that $\|\Phi(x)\|(\partial M) \leq$ $\delta / 4$. Thus, for all $x, y \in X$,

$$
\mathbf{M}(\Phi(x)-\Phi(y)) \leq \mathbf{M}(\bar{\Phi}(x)-\bar{\Phi}(y))+\delta / 2 .
$$

Given $p \in M$ and $x \in X$, there is $r=r(p, x)>0$ and $U_{x} \subset X$ an open neighborhood of $x$ so that

$$
\|\Phi(y)\|\left(B_{r}(p)\right)<\delta \quad \text { for all } y \in U_{x} .
$$

By compactness, we can select a finite covering $\left\{B_{r_{k}}\left(p_{k}\right) \times U_{x_{k}}\right\}_{k=1}^{N}$ of $M \times X$, where $r_{k}=r\left(p_{k}, x_{k}\right) / 2$. If $\bar{r}=\min \left\{r_{k}\right\}_{k=1}^{N}$, then

$$
\|\Phi(x)\|\left(B_{\bar{r}}(p)\right)<\delta \text { for all }(p, x) \in M \times X
$$

and the result follows. 
The $p$-width of $M$ of dimension $k$ is

$$
\omega_{p}^{k}(M)=\inf _{\Phi \in \mathcal{P}_{p}^{k}(M)} \sup \{\mathbf{M}(\Phi(x)): x \in \operatorname{dmn}(\Phi)\},
$$

where $\operatorname{dmn}(\Phi)$ is the domain of $\Phi$. We also set

$$
\tilde{\omega}_{p}^{k}(M)=p^{-\frac{n+1-k}{n+1}} \omega_{p}^{k}(M) .
$$

Gromov and Guth [5, 9] studied the asymptotic behaviour of $\omega_{p}^{k}(M)$ as $p \rightarrow \infty$ and showed:

2.8. Theorem. There is $C_{k, M}>0$ such that

$$
C_{k, M}^{-1} \leq \tilde{\omega}_{p}^{k}(M) \leq C_{k, M} \quad \text { for all } p \in \mathbb{N} \text {. }
$$

2.9. Approximation results. Given $X$ a cubical subcomplex, $l \in \mathbb{N}$, an integer $0 \leq k<n+1$, and a map $\phi: X(l)_{0} \rightarrow \mathcal{Z}_{k \text {,rel }}\left(M, \partial M ; \mathbb{Z}_{2}\right)$, we define the fineness of $\phi$ to be

$$
\mathbf{f}(\phi)=\sup \left\{\mathbf{M}(\phi(x)-\phi(y)): x, y \text { adjacent vertices in } X(l)_{0}\right\} .
$$

2.10. Theorem. Let $\Phi: X \rightarrow \mathcal{Z}_{k, \text { rel }}\left(M, \partial M ; \mathbb{Z}_{2}\right)$ be a continuous map in the flat topology that has no concentration of mass. There exist a sequence of maps

$$
\phi_{i}: X\left(l_{i}\right)_{0} \rightarrow \mathcal{Z}_{k, \text { rel }}\left(M, \partial M ; \mathbb{Z}_{2}\right), \quad i \in \mathbb{N}
$$

with $l_{i}<l_{i+1}$, and a sequence of positive numbers $\left\{\delta_{i}\right\}_{i \in \mathbb{N}}$ converging to zero such that for all $i \in \mathbb{N} \mathbf{f}\left(\phi_{i}\right)<\delta_{i}$,

$$
\sup \left\{\mathcal{F}\left(\phi_{i}(x)-\Phi(x)\right): x \in X\left(l_{i}\right)_{0}\right\} \leq \delta_{i},
$$

and

$$
\sup \left\{\mathbf{M}\left(\phi_{i}(x)\right): x \in X\left(l_{i}\right)_{0}\right\} \leq \sup \{\mathbf{M}(\Phi(x)): x \in X\}+\delta_{i} .
$$

When $k=n$ and $\partial M=\emptyset$, the result was proven in Theorem 13.1 in [15]. We leave the proof of Theorem 2.10 to Appendix A.

The purpose of the next theorem is to construct a continuous map in the mass norm out of a discrete map with small fineness.

2.11. Theorem. There exist positive constants $C_{0}=C_{0}(M, m) \geq 1$ and $\delta_{0}=\delta_{0}(M)$ so that if $Y$ is a cubical subcomplex of $I(m, l)$ and

$$
\phi: Y_{0} \rightarrow \mathcal{Z}_{k, \text { rel }}\left(M, \partial M ; \mathbb{Z}_{2}\right)
$$

has $\mathbf{f}(\phi)<\delta_{0}$, then there exists a map

$$
\Phi: Y \rightarrow \mathcal{Z}_{k, \text { rel }}\left(M, \partial M ; \mathbf{M} ; \mathbb{Z}_{2}\right)
$$

continuous in the mass norm and satisfying

(i) $\Phi(x)=\phi(x)$ for all $x \in Y_{0}$;

(ii) if $\alpha$ is some $j$-cell in $Y_{j}$, then $\Phi$ restricted to $\alpha$ depends only on the values of $\phi$ assumed on the vertices of $\alpha$; 
(iii)

$$
\sup \{\mathbf{M}(\Phi(x)-\Phi(y)): x, y \text { lie in a common cell of } Y\} \leq C_{0} \mathbf{f}(\phi) .
$$

The map $\Phi$ is called the Almgren extension of $\phi$. We postpone its proof to Appendix B because a similar result was proven in Theorem 14.1 of [15].

The proof of Proposition 3.5 of [16] can be extended in a straightforward way to show that

2.12. Proposition. Let $Y$ be a cubical subcomplex of $I(m, l)$. There exists $\eta=\eta(M, m)>0$ with the following property:

If $\Phi_{1}, \Phi_{2}: Y \rightarrow \mathcal{Z}_{k, \text { rel }}\left(M, \partial M ; \mathbb{Z}_{2}\right)$ are continuous maps in the flat topology such that

$$
\sup \left\{\mathcal{F}\left(\Phi_{1}(y), \Phi_{2}(y)\right): y \in Y\right\}<\eta,
$$

then $\Phi_{1}$ is homotopic to $\Phi_{2}$ in the flat topology.

The previous results have the following corollary.

2.13. Corollary. Given $\Phi \in \mathcal{P}_{p}^{k}(M)$ there is a sequence of positive numbers $\left\{\delta_{i}\right\}_{i \in \mathbb{N}}$ converging to zero and a sequence $\left\{\Phi_{i}\right\}_{i \in \mathbb{N}} \subset \mathcal{P}_{p}^{k}(M)$ of maps continuous in the mass topology such that, for all $i \in \mathbb{N}, \operatorname{dmn}(\Phi)=\operatorname{dmn}\left(\Phi_{i}\right)$ and

$$
\sup _{x \in \operatorname{dmn}(\Phi)}\left\{\mathbf{M}\left(\Phi_{i}(x)\right)\right\} \leq \sup _{x \in \operatorname{dmn}(\Phi)}\{\mathbf{M}(\Phi(x))\}+\delta_{i}
$$

Thus, when computing $\omega_{p}^{k}(M)$, we can consider only the maps in $\mathcal{P}_{p}^{k}(M)$ that are continuous in the mass topology.

Proof. From Theorem 2.10 and Theorem 2.11 we obtain a sequence $\left\{\Phi_{i}\right\}_{i \in \mathbb{N}}$ of maps continuous in the mass topology with $\operatorname{dmn}(\Phi)=\operatorname{dmn}\left(\Phi_{i}\right)$ for all $i \in \mathbb{N}$ and a sequence $\left\{\delta_{i}\right\}_{i \in \mathbb{N}}$ tending to zero such that

$$
\sup _{x \in \operatorname{dmn}(\Phi)}\left\{\mathbf{M}\left(\Phi_{i}(x)\right)\right\} \leq \sup _{x \in \operatorname{dmn}(\Phi)}\{\mathbf{M}(\Phi(x))\}+\delta_{i}
$$

and

$$
\sup _{x \in \operatorname{dmn}(\Phi)}\left\{\mathcal{F}\left(\Phi_{i}(x)-\Phi(x)\right)\right\} \leq \delta_{i}
$$

The corollary follows from Proposition 2.12.

2.14. Restriction of currents. Let $R \subset \Omega$ be two Lipschitz domains. Given $T \in \mathcal{Z}_{k \text {,rel }}\left(\Omega, \partial \Omega ; \mathbb{Z}_{2}\right)$ it is not necessarily true that $T\llcorner R$ belongs to $\mathcal{Z}_{k, \text { rel }}\left(R, \partial R ; \mathbb{Z}_{2}\right)$ because its boundary might have unbounded mass. Thus the following lemma is needed. The proof is a bit technical and thus could be skipped in a first reading. 
2.15. Lemma. Let $R \subset \Omega$ be two Lipschitz domains. Consider $0 \leq k<$ $n+1, p \in \mathbb{N}$, and $\Phi \in \mathcal{P}_{p}^{k}(\Omega)$ continuous in the mass topology with $X:=$ $\operatorname{dmn}(\Phi)$.

(1) For any $\varepsilon>0$ there is

$$
\hat{\Phi}: X \rightarrow \mathcal{Z}_{k, \text { rel }}\left(R, \partial R ; \mathbb{Z}_{2}\right)
$$

continuous in the mass topology such that

$$
\mathbf{M}(\hat{\Phi}(x)) \leq(1+\varepsilon)^{k} \mathbf{M}(\Phi(x)\llcorner R) \text { for all } x \in X
$$

and $\hat{\Phi}^{*} \bar{\lambda}_{k}=\Phi^{*} \bar{\lambda}_{k}$.

(2) For any $\bar{\varepsilon}>0$ and integer $0 \leq q \leq p$, the open set

$$
\left\{x \in X: \mathbf{M}\left(\Phi(x)\llcorner R)<\omega_{q}^{k}(R)-\bar{\varepsilon}\right\}\right.
$$

is contained in an open set $U$ such that the pull back of $\lambda_{k}=\Phi^{*} \bar{\lambda}_{k}$ by the inclusion map $\iota: U \rightarrow X$ has its q-th cup power vanishing in $H^{q(n+1-k)}\left(U ; \mathbb{Z}_{2}\right)$.

Proof. We start with some discussion regarding the domain $R$. In Theorem 2.7 and Proposition 2.3 of [11] it is shown the existence of a smooth vector field $Y$ in $\mathbb{R}^{n+1}$ with $|Y|=1$ on $\partial R$ and $\kappa>0$ such that $Y . \nu \geq \kappa$ a.e. on $\partial R$, where $\nu$ denotes the measure theoretic inward unit normal of $\partial R$.

Given $t \in \mathbb{R}$ set

$$
F_{t}: R \rightarrow \mathbb{R}^{n+1} \quad F_{t}(x)=x+t Y(x) .
$$

From [11, Proposition 4.15 and Theorem 4.19] we have the existence of $t_{0}$ so that, for all $0<t \leq t_{0}, F_{t}$ is a bi-Lipschitz map and $R_{t}:=F_{t}(R)$ is a Lipschitz domain contained in the interior of $R$. From $[11,(4.67)]$ it also follows the existence of a Lipschitz function $u$ defined on $\mathbb{R}^{n+1}$ with Lipschitz constant bounded by $C$ such that $R_{t}=\{x: u(x) \geq t\}$ for all $0 \leq t \leq t_{0}$. Furthermore, we can also assume that

$$
\left|D F_{t}^{-1}\right|(x) \leq(1+\varepsilon) \quad \text { for all } x \in \Omega \text {. }
$$

Let $L=\sup _{x \in X}\{\mathbf{M}(\Phi(x))\}$. For all $l \in \mathbb{N}$ we can find $0<s(l)<t_{0}$ so that (see [21, Section 28])

(a) $\Phi(x)\left\llcorner R_{s(l)} \in \mathcal{Z}_{k, \text { rel }}\left(R_{s(l)}, \partial R_{s(l)} ; \mathbb{Z}_{2}\right)\right.$ for all $x \in X(l)_{0}$;

(b) $\mathbf{M}\left(\partial\left(\Phi(x)\left\llcorner R_{s(l)}\right)\right) \leq C L t_{0}^{-1}\right.$ for all $x \in X(l)_{0}$.

Set

$$
\phi_{l}: X(l)_{0} \rightarrow \mathcal{Z}_{k, \mathrm{rel}}\left(R, \partial R ; \mathbb{Z}_{2}\right), \quad \phi_{l}(x)=\left(F_{s(l)}^{-1}\right)_{\#}\left(\Phi(x)\left\llcorner R_{s(l)}\right) .\right.
$$

We have for all $x, y \in X(l)_{0}$

$$
\mathbf{M}\left(\phi_{l}(x)\right) \leq(1+\varepsilon)^{k} \mathbf{M}\left(\Phi(x)\left\llcorner R_{s(l)}\right) \leq(1+\varepsilon)^{k} \mathbf{M}(\Phi(x)\llcorner R)\right.
$$

and

$$
\mathbf{M}\left(\phi_{l}(x)-\phi_{l}(y)\right) \leq(1+\varepsilon)^{k} \mathbf{M}(\Phi(x)-\Phi(y)) .
$$


Moreover

$$
\sup _{x \in X}\left\{\mathbf{M}\left(\phi_{l}(x)\right)+\mathbf{M}\left(\partial\left(\phi_{l}(x)\right)\right)\right\} \leq(1+\varepsilon)^{k}\left(L+C L t_{0}^{-1}\right)
$$

and so we can apply Federer-Fleming Compactness Theorem and a diagonalization argument to conclude the existence of a subsequence $\left\{\phi_{l_{i}}\right\}_{i \in \mathbb{N}}$ such that $\phi_{l_{i}}(x)$ converges for all $x \in X(l)_{0}, l \in \mathbb{N}$. Using (8) we obtain a continuous function in the mass topology

$$
\hat{\Phi}: X \rightarrow \mathcal{Z}_{k, \text { rel }}\left(R, \partial R ; \mathbb{Z}_{2}\right)
$$

such that

$$
\mathbf{M}(\hat{\Phi}(x)) \leq(1+\varepsilon)^{k} \mathbf{M}(\Phi(x)\llcorner R) \text { for all } x \in X .
$$

For all $i \in \mathbb{N}$ large enough the Almgren extension $\Phi_{i}$ of $\phi_{l_{i}}$ is well defined and homotopic in the flat topology to $\hat{\Phi}$. Choose such $i \in \mathbb{N}$. To complete the proof of Lemma 2.15 (1) we need to show that $\Phi_{i}^{*} \bar{\lambda}_{k}=\Phi^{*} \bar{\lambda}_{k}$.

An element in $H_{n+1-k}\left(X ; \mathbb{Z}_{2}\right)$ is represented by a continuous map $\tau: Y \rightarrow$ $X$, where $Y$ is a $(n+1-k)$-cubical subcomplex of $I\left(m^{\prime}, j\right)$ and $\partial Y=0$.

Choose $i \in \mathbb{N}$ large enough so that for every $x, y$ belonging to a common cell of $X\left(l_{i}\right)$ we have

$$
\mathbf{M}(\Phi(x)-\Phi(y))<\min \left\{\nu_{\Omega, \partial \Omega}, \frac{\delta_{0}}{(1+\varepsilon)^{k}}, \frac{\nu_{R, \partial R}}{C_{0}(1+\varepsilon)^{k}}, \frac{\eta}{5 C_{0}(1+\varepsilon)^{k}}\right\}
$$

where $\nu_{\Omega, \partial \Omega}, \nu_{R, \partial R}$ are given in $(5), \delta_{0}=\delta_{0}(R), C_{0}=C_{0}\left(R, m^{\prime}\right)$ are given by Theorem 2.11, and $\eta=\eta\left(R, m^{\prime}\right)$ is given by Proposition 2.12.

Choose $t_{1}$ with $s\left(l_{i}\right)<t_{1}<t_{0}$ and such that for all $x \in X\left(l_{i}\right)_{0}$

$$
\|\Phi(x)\|\left(R_{s\left(l_{i}\right)} \backslash R_{t_{1}}\right)<\frac{\eta}{(1+\varepsilon)^{k}}
$$

and

$$
\mathcal{F}\left(( F _ { s ( l _ { i } ) } ^ { - 1 } ) _ { \# } \left(\Phi(x)\left\llcorner R_{s\left(l_{i}\right)}\right),\left(F_{t}^{-1}\right)_{\#}\left(\Phi(x)\left\llcorner R_{s\left(l_{i}\right)}\right)\right)<\frac{\eta}{5}\right.\right.
$$

for all $s\left(l_{i}\right) \leq t \leq t_{1}$. The choice in (10) is possible because for all $x \in X\left(l_{i}\right)_{0}$ we have that $\|\Phi(x)\|\left(\partial R_{s\left(l_{i}\right)}\right)=0$, and the choice in (11) is possible due to the homotopy formula [21, Section 26.22] .

Without loss of generality we can assume that for every cell $\alpha \in Y, \tau(\alpha)$ is contained in a cell in $X\left(l_{i}\right)_{0}$.

Set $\Psi=\Phi \circ \tau$ and $\Psi_{i}=\Phi_{i} \circ \tau$. These maps represent elements $\sigma$ and $\hat{\sigma}$ in $H_{n+1-k}\left(\mathcal{Z}_{k, \text { rel }}\left(\Omega, \partial \Omega ; \mathbb{Z}_{2}\right) ; \mathbb{Z}_{2}\right)$ and $H_{n+1-k}\left(\mathcal{Z}_{k, \text { rel }}\left(R, \partial R ; \mathbb{Z}_{2}\right) ; \mathbb{Z}_{2}\right)$ respectively.

From (9) we see that the map $\Psi$ satisfies (5) (with $l=0$ ) and so we obtain a chain map of degree $k$ given by $\psi: Y \rightarrow \mathbf{I}_{*}\left(\Omega ; \mathbb{Z}_{2}\right)$. Using the slicing theory of [21, Section 28] we find $s\left(l_{i}\right)<t<t_{1}$ such that

- $\psi(x)\left\llcorner R_{t} \in \mathcal{Z}_{k \text {,rel }}\left(R_{t}, \partial R_{t} ; \mathbb{Z}_{2}\right)\right.$ for all $x \in Y_{0}$;

- for every cell $\alpha \in Y_{p}$ we have $\psi(\alpha)\left\llcorner R_{t} \in \mathbf{I}_{p+k}\left(R_{t} ; \mathbb{Z}_{2}\right)\right.$. 
We consider the chain map of degree $k$

$$
\bar{\psi}: Y \rightarrow \mathbf{I}_{*}\left(R ; \mathbb{Z}_{2}\right), \quad \bar{\psi}(\alpha)=\left(F_{t}^{-1}\right)_{\#}\left(\psi(\alpha)\left\llcorner R_{t}\right) .\right.
$$

From (9) we see that $\bar{\psi}_{\mid Y_{0}}$ satisfies the conditions of Theorem 2.11 and we denote by $\bar{\Psi}$ its Almgren extension.

From (9) we have that for all $x \in Y$, there are $x^{\prime} \in X\left(l_{i}\right)_{0}$ and $x^{\prime \prime} \in X$ belonging to a common cell of $X\left(l_{i}\right)$ and such that

$$
\mathcal{F}\left(\Psi_{i}(x), \bar{\Psi}(x)\right)<\mathcal{F}\left(( F _ { s ( l _ { i } ) } ^ { - 1 } ) _ { \# } \left(\Phi\left(x^{\prime}\right)\left\llcorner R_{s\left(l_{i}\right)}\right),\left(F_{t}^{-1}\right)_{\#}\left(\Phi\left(x^{\prime \prime}\right)\left\llcorner R_{t}\right)\right)+\frac{2}{5} \eta .\right.\right.
$$

Hence we obtain from (9), (10), and (11) that

$$
\mathcal{F}(\tilde{\Psi}(x), \bar{\Psi}(x))<\eta \quad \text { for all } x \in Y
$$

and Proposition 2.12 implies that $\Psi_{i}$ and $\bar{\Psi}$ are homotopic in the flat topology. Hence they represent the same element $\hat{\sigma}$ in homology.

From (9) we see that the map $\bar{\Psi}$ satisfies (5) (with $l=0$ ). From (6) and the definition of $\bar{\psi}$ we have that $[\psi]\left\llcorner R=[\bar{\psi}]\right.$ and so $\bar{\lambda}_{k}(\sigma)=\bar{\lambda}_{k}(\hat{\sigma})$, which is what we wanted to show.

We now prove Lemma 2.15 (2). Choose $\varepsilon$ so that

$$
(1+\varepsilon)^{k} \omega_{q}^{k}(R)<\omega_{q}^{k}(R)-\bar{\varepsilon} / 2 .
$$

Considering $\hat{\Phi}$ given by Lemma 2.15 (1) we have that

$$
\left\{x \in X: \mathbf{M}\left(\Phi(x)\llcorner R)<\omega_{q}^{k}(R)-\varepsilon\right\} \subset\left\{x \in X: \mathbf{M}(\hat{\Phi}(x))<\omega_{q}^{k}(R)-\bar{\varepsilon} / 2\right\} .\right.
$$

Denote the set on the right by $U$ and we assume without loss of generality that its closure $\bar{U}$ is a cubical complex. If Lemma 2.15 (2) did not hold then $\hat{\Phi}_{\mid \bar{U}} \in \mathcal{P}_{q}^{k}(R)$ and this contradicts the definition of $\omega_{q}^{k}(R)$.

\section{WEYL LAW FOR DOMAINS}

In what follows $C$ denotes the unit cube in $\mathbb{R}^{n+1}$. Two regions of $\mathbb{R}^{n+1}$ are said to be similar if they differ by an isometry and scaling. Given a real number, $[x]$ denotes its integer part. Recall the definition of $\tilde{\omega}_{p}^{k}$ in (7). All domains considered are assumed to satisfy the topological condition (4).

3.1. Lusternick-Schnirelman Inequality. Fix $0 \leq k<n+1$ and consider Lipschitz domains $\Omega_{0},\left\{\Omega_{i}\right\}_{i=1}^{N},\left\{\Omega_{i}^{*}\right\}_{i=1}^{N}$ such that

- $\Omega_{i}$ have unit volume for all $i=0, \ldots, N$;

- $\Omega_{i}^{*}$ is similar to $\Omega_{i}$ for all $i=1, \ldots, N$;

- $\Omega_{i}^{*} \subset \Omega_{0}$ for all $i=1, \ldots, N$ and the interiors of $\left\{\Omega_{i}^{*}\right\}_{i=1}^{N}$ are pairwise disjoint.

There is a constant $c=c\left(\Omega_{0}, k, n\right)$ such that, with $V=\min \left\{\operatorname{vol}\left(\Omega_{i}^{*}\right)\right\}_{i=1}^{N}$ and $p_{i}=\left[p \operatorname{vol}\left(\Omega_{i}^{*}\right)\right], i=1, \ldots, N$, we have for all $p \in \mathbb{N}$

$$
\tilde{\omega}_{p}^{k}\left(\Omega_{0}\right) \geq \sum_{i=1}^{N} \operatorname{vol}\left(\Omega_{i}^{*}\right) \tilde{\omega}_{p_{i}}^{k}\left(\Omega_{i}\right)-\frac{c}{p V} .
$$


Proof. Set

$$
\bar{p}=\sum_{i=1}^{N} p_{i}=\sum_{i=1}^{N}\left[p \operatorname{vol}\left(\Omega_{i}^{*}\right)\right] \leq p \operatorname{vol}\left(\Omega_{0}\right)=p .
$$

Given $\Phi \in \mathcal{P}_{p}^{k}\left(\Omega_{0}\right)$ continuous in the mass topology (with $X=\operatorname{dmn}(\Phi)$ ) and $\varepsilon>0$, for each $i=1, \ldots, N$ consider the open set $U_{i}$ given by Lemma 2.15 (2) that contains the open set

$$
\left\{x \in X: \mathbf{M}\left(\Phi(x)\left\llcorner\Omega_{i}^{*}\right)<\omega_{p_{i}}^{k}\left(\Omega_{i}^{*}\right)-\varepsilon / N\right\} .\right.
$$

Thus, denoting by $\iota_{i}: U_{i} \rightarrow X$ the inclusion maps, we have that $\left(\iota_{i}^{*} \lambda_{k}\right)^{p_{i}}=0$ in $H^{p_{i}(n+1-k)}\left(U_{i} ; \mathbb{Z}_{2}\right)$ for all $i=1, \ldots, N$, where $\lambda_{k}=\Phi^{*}\left(\bar{\lambda}_{k}\right)$.

For all $i=1, \ldots, N$, the exact sequence

$$
H^{p_{i}(n+1-k)}\left(X, U_{i} ; \mathbb{Z}_{2}\right) \stackrel{j^{*}}{\rightarrow} H^{p_{i}(n+1-k)}\left(X ; \mathbb{Z}_{2}\right) \stackrel{\iota_{i}^{*}}{\rightarrow} H^{p_{i}(n+1-k)}\left(U_{i} ; \mathbb{Z}_{2}\right)
$$

implies the existence of $\lambda_{i} \in H^{p_{i}(n+1-k)}\left(X, U_{i} ; \mathbb{Z}_{2}\right)$ so that $j^{*}\left(\lambda_{i}\right)=\lambda_{k}^{p_{i}}$. Therefore

$$
j^{*}\left(\lambda_{1}\right) \smile \ldots \smile j^{*}\left(\lambda_{N}\right)=\lambda_{k}^{p_{1}+\ldots+p_{N}} \neq 0 \text { in } H^{\bar{p}(n+1-k)}\left(X ; \mathbb{Z}_{2}\right),
$$

because $\lambda_{k}^{p} \neq 0$ and $\bar{p} \leq p$.

We now claim that $X \neq \cup_{i=1}^{N} U_{i}$. Indeed, if otherwise then

$$
H^{\bar{p}(n+1-k)}\left(X, U_{1} \cup \cdots \cup U_{N} ; \mathbb{Z}_{2}\right)=H^{\bar{p}(n+1-k)}\left(X, X ; \mathbb{Z}_{2}\right)=0
$$

and from the natural notion of relative cup product (see [10], p 209)

$$
\begin{aligned}
H^{p_{1}(n+1-k)}\left(X, U_{1} ; \mathbb{Z}_{2}\right) \smile \cdots \smile & H^{p_{N}(n+1-k)}\left(X, U_{N} ; \mathbb{Z}_{2}\right) \\
& \rightarrow H^{\bar{p}(n+1-k)}\left(X, U_{1} \cup \cdots \cup U_{N} ; \mathbb{Z}_{2}\right)=0
\end{aligned}
$$

we see that $\lambda_{1} \smile \ldots \smile \lambda_{N}=0$ which means that

$$
\lambda^{\bar{p}}=j^{*}\left(\lambda_{1}\right) \smile \ldots \smile j^{*}\left(\lambda_{N}\right)=j^{*}\left(\lambda_{1} \smile \ldots \smile \lambda_{N}\right)=0 .
$$

This proves the claim.

Thus there is $x \in X \backslash \cup_{i=1}^{N} U_{i}$ and so $\mathbf{M}(\Phi(x)) \geq \sum_{i=1}^{N} \omega_{p_{i}}^{k}\left(\Omega_{i}^{*}\right)-\varepsilon$. Using Corollary 2.13 and then making $\varepsilon$ tend to zero we obtain

$$
\omega_{p}^{k}\left(\Omega_{0}\right) \geq \sum_{i=1}^{N} \omega_{p_{i}}^{k}\left(\Omega_{i}^{*}\right) .
$$


As a result there is a constant $b=b(n, k)$ such that, using Theorem 2.8,

$$
\begin{aligned}
\tilde{\omega}_{p}^{k}\left(\Omega_{0}\right) & =p^{-\frac{n+1-k}{n+1}} \omega_{p}^{k}\left(\Omega_{0}\right) \geq p^{-\frac{n+1-k}{n+1}} \sum_{i=1}^{N} \omega_{p_{i}}^{k}\left(\Omega_{i}^{*}\right) \\
& =p^{-\frac{n+1-k}{n+1}} \sum_{i=1}^{N} \operatorname{vol}\left(\Omega_{i}^{*}\right)^{\frac{k}{n+1}} \omega_{p_{i}}^{k}\left(\Omega_{i}\right) \\
& =\sum_{i=1}^{N} \operatorname{vol}\left(\Omega_{i}^{*}\right)\left(\frac{p_{i}}{p \operatorname{vol}\left(\Omega_{i}^{*}\right)}\right)^{\frac{n+1-k}{n+1}} \tilde{\omega}_{p_{i}}^{k}\left(\Omega_{i}\right) \\
& \geq \sum_{i=1}^{N} \operatorname{vol}\left(\Omega_{i}^{*}\right)\left(1-\frac{1}{p \operatorname{vol}\left(\Omega_{i}^{*}\right)}\right)^{\frac{n+1-k}{n+1}} \tilde{\omega}_{p_{i}}^{k}\left(\Omega_{i}\right) \\
& \geq \sum_{i=1}^{N} \operatorname{vol}\left(\Omega_{i}^{*}\right) \tilde{\omega}_{p_{i}}^{k}\left(\Omega_{i}\right)-\frac{b}{p V} \sum_{i=1}^{N} \operatorname{vol}\left(\Omega_{i}^{*}\right) \tilde{\omega}_{p_{i}}^{k}\left(\Omega_{i}\right) \\
\geq & \sum_{i=1}^{N} \operatorname{vol}\left(\Omega_{i}^{*}\right) \tilde{\omega}_{p_{i}}^{k}\left(\Omega_{i}\right)-\frac{b C_{k}}{p V} \sum_{i=1}^{N} \operatorname{vol}\left(\Omega_{i}^{*}\right) \\
\geq & \sum_{i=1}^{N} \operatorname{vol}\left(\Omega_{i}^{*}\right) \tilde{\omega}_{p_{i}}^{k}\left(\Omega_{i}\right)-\frac{b C_{k}}{p V} .
\end{aligned}
$$

We can now prove the main theorem of this section

3.2. Weyl Law for domains. For all $0 \leq k<n+1$, there exists a constant $a(n, k)$ such that, for every Lipschitz domain $\Omega$ satisfying (4), we have

$$
\lim _{p \rightarrow \infty} \omega_{p}^{k}(\Omega) p^{-\frac{n+1-k}{n+1}}=a(n, k) \operatorname{vol}(\Omega)^{\frac{k}{n+1}} .
$$

Proof. Without loss of generality we assume that $\Omega$ has unit volume. We start with the following lemma:

3.3. Lemma. $\liminf \inf _{p \rightarrow \infty} \tilde{\omega}_{p}^{k}(C)=\lim \sup _{p \rightarrow \infty} \tilde{\omega}_{p}^{k}(C)$.

Proof. Choose $\left\{p_{l}\right\}_{l \in \mathbb{N}},\left\{q_{j}\right\}_{j \in \mathbb{N}}$ so that

$$
\limsup _{p \rightarrow \infty} \tilde{\omega}_{p}^{k}(C)=\lim _{l \rightarrow \infty} \tilde{\omega}_{p_{l}}^{k}(C) \text { and } \liminf _{p \rightarrow \infty} \tilde{\omega}_{p}^{k}(C)=\lim _{j \rightarrow \infty} \tilde{\omega}_{q_{j}}^{k}(C) .
$$

With $l$ fixed and for all $j$ large enough so that $\delta_{j}:=p_{l} / q_{j}<1$, consider $N_{j}$ to be the maximum number of cubes $\left\{C_{i}^{*}\right\}_{i=1}^{N_{j}}, \operatorname{vol}\left(C_{i}^{*}\right)=\delta_{j}$ for all $i$, with pairwise disjoint interiors contained in $C$. We must have $\delta_{j} N_{j} \rightarrow 1$ as $j \rightarrow \infty$. 
From the Lusternick-Schnirelman Inequality 3.1 we obtain

$$
\tilde{\omega}_{q_{j}}^{k}(C) \geq \sum_{i=1}^{N_{j}} \operatorname{vol}\left(C_{i}^{*}\right) \tilde{\omega}_{p_{l}}^{k}(C)+O\left(p_{l}^{-1}\right)=\delta_{j} N_{j} \tilde{\omega}_{p_{l}}^{k}(C)+O\left(p_{l}^{-1}\right) .
$$

Making $j \rightarrow \infty$ and then $l \rightarrow \infty$ we obtain

$$
\liminf _{p \rightarrow \infty} \tilde{\omega}_{p}^{k}(C) \geq \limsup _{p \rightarrow \infty} \tilde{\omega}_{p}^{k}(C)
$$

Set $a(n, k)=\lim _{p \rightarrow \infty} \tilde{\omega}_{p}^{k}(C)$.

3.4. Lemma. $\liminf \operatorname{in}_{p \rightarrow \infty} \tilde{\omega}_{p}^{k}(\Omega) \geq a(n, k)$.

Proof. Given any $\varepsilon>0$, one can find a family of cubes $\left\{C_{i}^{*}\right\}_{i=1}^{N}$ with pairwise disjoint interiors contained in $\Omega$, all with the same volume $\delta_{i}$, and such that

$$
\sum_{i=1}^{N} \operatorname{vol}\left(C_{i}^{*}\right) \geq 1-\varepsilon
$$

From the Lusternick-Schnirelman Inequality 3.1 we obtain

$$
\tilde{\omega}_{p}^{k}(\Omega) \geq \sum_{i=1}^{N} \operatorname{vol}\left(C_{i}^{*}\right) \tilde{\omega}_{\left[p \operatorname{vol}\left(C_{i}^{*}\right)\right]}^{k}(C)-\frac{c}{p \delta_{i}}
$$

and thus making $p \rightarrow \infty$ we have

$$
\liminf _{p \rightarrow \infty} \tilde{\omega}_{p}^{k}(\Omega) \geq(1-\varepsilon) \liminf _{p \rightarrow \infty} \tilde{\omega}_{p}^{k}(C)=(1-\varepsilon) a(n, k) .
$$

The result follows from the arbitrariness of $\varepsilon$.

3.5. Lemma. There are regions $\left\{\Omega_{i}^{*}\right\}_{i \in \mathbb{N}}$ contained in $C$, with pairwise disjoint interior, all similar to $\Omega$, and such that for all $\varepsilon>0$ we can choose $N \in \mathbb{N}$ so that $\sum_{i=1}^{N} \operatorname{vol}\left(\Omega_{i}^{*}\right) \geq 1-\varepsilon$.

Proof. Choose $\Omega_{1}$ contained in the interior of $C$, similar to $\Omega$, and denote its volume by $v$. Set $R_{1}$ to be the closure $C \backslash \Omega_{1}$ and find cubes $\left\{C_{i, 1}\right\}_{i=1}^{Q_{1}}$ contained in $R_{1}$ with pairwise disjoint interiors, and such that $\sum_{i=1}^{Q_{1}} \operatorname{vol}\left(C_{i, 1}\right) \geq \operatorname{vol}\left(R_{1}\right) / 2$. This is possible because $R_{1}$ is a Lipschitz domain. For all $i=1, \ldots, Q_{1}$, let $\Omega_{i, 1}$ be a region similar to $\Omega_{1}$, contained in the interior of $C_{i, 1}$, and with volume $v \operatorname{vol}\left(C_{i, 1}\right)$.

Next, set $\Omega_{2}=\cup_{i=1}^{Q_{1}} \Omega_{i, 1}$ and consider $R_{2}$ to be the closure of $C \backslash\left(\Omega_{1} \cup \Omega_{2}\right)$. Again, find cubes $\left\{C_{i, 2}\right\}_{i=1}^{Q_{2}}$ contained in $R_{2}$, with pairwise disjoint interiors, and such that $\sum_{i=1}^{Q_{2}} \operatorname{vol}\left(C_{i, 2}\right) \geq \operatorname{vol}\left(R_{2}\right) / 2$. For all $i=1, \ldots, Q_{2}$, let $\Omega_{i, 2}$ be a region similar to $\Omega_{1}$, contained in the interior of $C_{i, 2}$, and with volume $v \operatorname{vol}\left(C_{i, 2}\right)$. Define $\Omega_{3}=\cup_{i=1}^{Q_{2}} \Omega_{i, 2}$ and proceed inductively. 
It suffices to check that $\alpha_{N}:=\sum_{j=1}^{N} \operatorname{vol}\left(\Omega_{j}\right)$ tends to 1 as $N$ tends to infinity. Indeed from the construction we have

$\alpha_{N+1} \geq \alpha_{N}(1-v / 2)+v / 2 \Longrightarrow \alpha_{N+1} \geq v / 2 \sum_{j=0}^{N-1}(1-v / 2)^{j}=1-(1-v / 2)^{N}$.

3.6. Lemma. $a(n, k) \geq \lim \sup _{p \rightarrow \infty} \tilde{\omega}_{p}^{k}(\Omega)$.

Proof. Given $\varepsilon>0$, choose $\left\{q_{l}\right\}_{l \in \mathbb{N}}$ so that

$$
\beta_{k}:=\limsup _{p \rightarrow \infty} \tilde{\omega}_{p}^{k}(\Omega)=\lim _{l \rightarrow \infty} \tilde{\omega}_{q_{l}}^{k}(\Omega) .
$$

Consider the collection of regions $\left\{\Omega_{i}^{*}\right\}_{i=1}^{N}$ given by the previous lemma. With $l$ fixed and $p$ large, set $\delta_{p}=q_{l} /\left(p \operatorname{vol}\left(\Omega_{1}^{*}\right)\right)$ and let $Q_{p}$ be the maximum number of cubes $\left\{C_{j}^{*}\right\}_{j=1}^{Q_{p}}$ with pairwise disjoint interiors contained in $C$ where all have volume $\delta_{p}$. We have $\delta_{p} Q_{p}$ approaching 1 as $p \rightarrow \infty$.

For each $j=1, \ldots, Q_{p}$ we have regions $\left\{\Omega_{i, j}\right\}_{i=1}^{N}$ inside $C_{j}$, with pairwise disjoint interiors, all similar to $\Omega$, and such that

$$
\operatorname{vol}\left(\Omega_{i, j}\right)=\delta_{p} \operatorname{vol}\left(\Omega_{i}^{*}\right) \quad i=1, \ldots, N
$$

and thus, with $v=\min \left\{\operatorname{vol}\left(\Omega_{i}^{*}\right)\right\}_{i=1}^{N}$,

$$
\min \left\{\operatorname{vol}\left(\Omega_{i, j}\right): i=1, \ldots, N, j=1, \ldots, Q_{p}\right\}=\delta_{p} v .
$$

Set $p_{i}=\left[p \delta_{p} \operatorname{vol}\left(\Omega_{i}^{*}\right)\right], i=1, \ldots, N$. From the Lusternick-Schnirelman Inequality 3.1 we have

$$
\begin{aligned}
\tilde{\omega}_{p}^{k}(C) & \geq \sum_{j=1}^{Q_{p}} \operatorname{vol}\left(\Omega_{1, j}\right) \tilde{\omega}_{p_{1}}^{k}(\Omega)+\sum_{j \geq 1, i \geq 2} \operatorname{vol}\left(\Omega_{i, j}\right) \tilde{\omega}_{p_{i}}^{k}(\Omega)+O\left(\frac{1}{p \delta_{p} v}\right) \\
& =Q_{p} \delta_{p}\left(\operatorname{vol}\left(\Omega_{1}^{*}\right) \tilde{\omega}_{q_{l}}^{k}(\Omega)+\sum_{i=2}^{N} \operatorname{vol}\left(\Omega_{i}^{*}\right) \tilde{\omega}_{p_{i}}^{k}(\Omega)\right)+O\left(\frac{1}{p \delta_{p} v}\right)
\end{aligned}
$$

Making $p$ tend to infinity we obtain

$$
a(n, k) \geq \operatorname{vol}\left(\Omega_{1}^{*}\right) \tilde{\omega}_{q_{l}}^{k}(\Omega)+\sum_{i=2}^{N} \operatorname{vol}\left(\Omega_{i}^{*}\right) \tilde{\omega}_{\left[q_{l} \frac{\operatorname{vol}\left(\Omega_{i}^{*}\right)}{\operatorname{vol}\left(\Omega_{1}^{*}\right)}\right]}^{k}(\Omega)+O\left(\frac{\operatorname{vol}\left(\Omega_{1}^{*}\right)}{q_{l} v}\right) .
$$

Making $l \rightarrow \infty$ and using Lemma 3.4 we obtain that

$$
a(n, k) \geq \operatorname{vol}\left(\Omega_{1}^{*}\right) \beta_{k}+a(n, k) \sum_{i=2}^{N} \operatorname{vol}\left(\Omega_{i}^{*}\right) .
$$

Lemma 3.5 implies then that

$$
\left(\operatorname{vol}\left(\Omega_{1}^{*}\right)+\varepsilon\right) a(n, k) \geq \operatorname{vol}\left(\Omega_{1}^{*}\right) \beta_{k}=\operatorname{vol}\left(\Omega_{1}^{*}\right) \limsup _{p \rightarrow \infty} \tilde{\omega}_{p}^{k}(\Omega) .
$$

The result follows by making $\varepsilon \rightarrow 0$. 
The desired result is a consequence of Lemma 3.4, Lemma 3.3, and Lemma 3.6 .

\section{Weyl LAW FOR COMPACT MANifOldS}

We consider a compact Riemannian $(n+1)$-manifold $\left(M^{n+1}, g\right)$ isometrically embedded in $\mathbb{R}^{Q}$ with smooth boundary $\partial M$ and satisfying (4). Recall the definition of $\tilde{\omega}_{p}^{k}(M)$ in (7), that $C$ denotes the unit cube in $\mathbb{R}^{n+1}$, and that, for every integer $0 \leq k<n+1$, we set

$$
a(n, k)=\lim _{p \rightarrow \infty} \tilde{\omega}_{p}^{k}(C) .
$$

The geodesic ball in $(M, g)$ of radius $r$ centred at $p \in M$ is denoted by $\mathcal{B}_{r}(p)$.

4.1. Theorem. For every integer $0 \leq k<n+1$ we have

$$
\liminf _{p \rightarrow \infty} \omega_{p}^{k}(M) p^{-\frac{n+1-k}{n+1}} \geq a(n, k) \operatorname{vol}(M)^{\frac{k}{n+1}} .
$$

Proof. Without loss of generality we assume that $\operatorname{vol}(M)=1$.

Given $\varepsilon>0$ there is $\bar{r}>0$ so that for every $\mathcal{B}_{r}(p) \subset M \backslash \partial M$ with $r \leq \bar{r}$ and $p \in M$, the Euclidean metric $g_{0}$ induced on $\mathcal{B}_{r}(p)$ via Riemannian normal coordinates centered at $p$ is such that $(1+\varepsilon)^{-1 / 2} g \leq g_{0} \leq(1+\varepsilon)^{1 / 2} g$. Denoting by $\left|B_{r}(0)\right|$ the volume of the Euclidean ball $B_{r}(0)$ we have

$$
(1+\varepsilon)^{-(n+1)} \operatorname{vol}\left(\mathcal{B}_{r}(p)\right) \leq\left|B_{r}(0)\right| \leq(1+\varepsilon)^{n+1} \operatorname{vol}\left(\mathcal{B}_{r}(p)\right)
$$

and $\omega_{p}^{k}\left(\mathcal{B}_{r}(p)\right) \geq(1+\varepsilon)^{-k} \omega_{p}^{k}\left(B_{r}(0)\right)$ for all $p \in \mathbb{N}$.

Choose a collection of pairwise disjoint geodesic balls $\mathcal{B}_{i} \subset M \backslash \partial M$, $i=1, \ldots, N$, all with radius smaller than $\bar{r}$, and such that $\sum_{i=1}^{N} \operatorname{vol}\left(\mathcal{B}_{i}\right) \geq$ $(1+\varepsilon)^{-1}$.

Let $B$ denote the unit volume ball in $\mathbb{R}^{n+1}$ and $B_{i}$ denote a Euclidean ball with the same radius as $\mathcal{B}_{i}, i=1, \ldots, N$. Reasoning like in the proof of (12) in Lusternick-Schnirelman Inequality 3.1 we have that

$$
\omega_{p}^{k}(M) \geq \sum_{i=1}^{N} \omega_{\left[p \operatorname{vol}\left(\mathcal{B}_{i}\right)\right]}^{k}\left(\mathcal{B}_{i}\right)
$$

and so, with $p_{i}=\left[p \operatorname{vol}\left(\mathcal{B}_{i}\right)\right], i=1, \ldots, N$,

$$
\begin{aligned}
\tilde{\omega}_{p}^{k}(M) & \geq(1+\varepsilon)^{-k} \sum_{i=1}^{N}\left|B_{i}\right|\left(\frac{p_{i}}{p\left|B_{i}\right|}\right)^{\frac{n+1-k}{n+1}} \tilde{\omega}_{p_{i}}^{k}(B) \\
& \geq(1+\varepsilon)^{-k} \sum_{i=1}^{N}\left|B_{i}\right|\left(\frac{\operatorname{vol}\left(\mathcal{B}_{i}\right)}{\left|B_{i}\right|}-\frac{1}{p\left|B_{i}\right|}\right)^{\frac{n+1-k}{n+1}} \tilde{\omega}_{p_{i}}^{k}(B) \\
& \geq(1+\varepsilon)^{-(n+k+1)} \sum_{i=1}^{N} \operatorname{vol}\left(\mathcal{B}_{i}\right)\left(\frac{\operatorname{vol}\left(\mathcal{B}_{i}\right)}{\left|B_{i}\right|}-\frac{1}{p\left|B_{i}\right|}\right)^{\frac{n+1-k}{n+1}} \tilde{\omega}_{p_{i}}^{k}(B) .
\end{aligned}
$$


Making $p \rightarrow \infty$ and using Theorem 3.2 we obtain

$$
\liminf _{p \rightarrow \infty} \tilde{\omega}_{p}^{k}(M) \geq(1+\varepsilon)^{-2 n-2} a(n, k) \sum_{i=1}^{N} \operatorname{vol}\left(\mathcal{B}_{i}\right) \geq(1+\varepsilon)^{-2 n-3} a(n, k) .
$$

The desired result follows by making $\varepsilon$ tend to zero.

We focus on the case where $k=n$ and set $a(n)=a(n, n)$. We drop the subscript or superscript $k$ in the notation, which means that $\omega_{p}^{n}(M)$ becomes $\omega_{p}(M), \mathcal{P}_{p}^{n}(M)$ becomes $\mathcal{P}_{p}(M)$ and so on. Condition (4) means that $M$ is connected.

4.2. Weyl Law for compact manifolds. For every compact Riemannian manifold $\left(M^{n+1}, g\right)$ with (possibly empty) boundary, we have

$$
\lim _{p \rightarrow \infty} \omega_{p}(M) p^{-\frac{1}{n+1}}=a(n) \operatorname{vol}(M)^{\frac{n}{n+1}} .
$$

Proof. Recalling the discussion in Section 1.4, we start by decomposing $M$ into regions that are almost Euclidean (denoted by $\left\{\mathcal{C}_{i}\right\}_{i=1}^{N}$ ) and then use those regions to construct a connected region $\Omega$ in Euclidean space.

Given $\varepsilon>0$, consider $\bar{r}$ so that for all $p \in M$ the ball $\mathcal{B}_{\bar{r}}(p)$ is $(1+\varepsilon / 2)$ bilipschitz diffeomorphic to some ball of radius $\bar{r}$ in the closed upper halfspace $\mathbb{R}^{n} \times \mathbb{R}_{+}$with the Euclidean metric. Choose a covering $\left\{\mathcal{B}_{i}\right\}_{i=1}^{\tilde{N}}$ of $M$ by balls of radius $\bar{r}$, so that balls of half the radius still cover $M$.

We now define a collection $\mathcal{C}=\left\{\mathcal{C}_{i}\right\}_{i=1}^{N}$ of domains with the following properties for all $i=1, \ldots, N$ :

- Each $\mathcal{C}_{i}$ is $(1+\varepsilon / 2)$-bilipschitz diffeomorphic to a Lipschitz domain in $\mathbb{R}^{n+1}$ with Euclidean metric;

- $\mathcal{C}$ is a covering of $M$;

- $\mathcal{C}_{i}$ 's have mutually disjoint interiors.

We first define domains $\tilde{\mathcal{C}}_{i}, i=1, \ldots, \tilde{N}$, inductively. We set $\tilde{\mathcal{C}}_{1}=\mathcal{B}_{1}$. For $i>1$ we set $\tilde{\mathcal{B}}_{i}$ to be a concentric ball in $\mathcal{B}_{i}$ of radius $r_{i} \in\left[\frac{\bar{r}}{2}, \bar{r}\right]$, so that the boundary of $\mathcal{B}_{i}$ intersects the boundaries of $\tilde{\mathcal{C}}_{1}, \ldots, \tilde{\mathcal{C}}_{i-1}$ transversally. We define $\tilde{\mathcal{C}}_{i}$ to be the closure of $\tilde{\mathcal{B}}_{i} \cap\left(M \backslash \cup_{j=1}^{i-1} \tilde{\mathcal{C}}_{j}\right)$. The transversality condition ensures that $\tilde{\mathcal{C}}_{i}$ is a Lipschitz domain for all $i=1, \ldots, \tilde{N}$. The collection $\mathcal{C}$ is formed by considering the connected components $\mathcal{C}_{1}, \ldots, \mathcal{C}_{N}$ of the domains $\tilde{\mathcal{C}}_{1}, \ldots, \tilde{\mathcal{C}}_{\tilde{N}}$.

For each $i=1, \ldots, N$, let $C_{i} \subset \mathbb{R}^{n+1}$ be a region $(1+\varepsilon / 2)$-bilipschitz diffeomorphic to $\mathcal{C}_{i}$. Consider a region $\Omega \subset \mathbb{R}^{n+1}$ that one obtains by connecting the $N$ disjoint regions $C_{i} \subset \mathbb{R}^{n+1}, i=1, \ldots, N$ consecutively by tubes of very small volume. The region $\Omega$ is connected and a Lipschitz domain. Moreover, making the volumes of the connecting tubes sufficiently small we obtain 


$$
\operatorname{vol}(\Omega) \leq(1+\varepsilon)^{n+1} \operatorname{vol}(M)
$$

Consider $\Phi \in \mathcal{P}_{p}(\Omega)$ continuous in the mass topology with $X=\operatorname{dmn}(\Phi)$. From Lemma 2.15 (i) we obtain, for all $i=1, \ldots, N, \Phi_{i} \in \mathcal{P}_{p}\left(C_{i}\right)$ with domain $X$,

$$
\mathbf{M}\left(\Phi_{i}(x)\right) \leq(1+\varepsilon)^{n} \mathbf{M}\left(\Phi(x)\left\llcorner C_{i}\right) \text { for all } x \in X,\right.
$$

and $\Phi_{i}^{*} \bar{\lambda}=\lambda$, where $\lambda=\Phi^{*} \bar{\lambda}$.

Next, we describe in general terms how to use the maps $\left\{\Phi_{i}\right\}_{i=1}^{N}$ to construct a $p$-sweepout of $M$. The elements $\Phi_{i}(x)$ have boundary in $\partial C_{i}$ and so one can choose $Z_{i}(x) \in \mathbf{I}_{n+1}\left(C_{i} ; \mathbb{Z}_{2}\right)$ so that the cycle $\partial Z_{i}(x)$ coincides with $\Phi_{i}(x)$ on the interior of $C_{i}$. Because the choice of $Z_{i}(x)$ is not unique $\left(C_{i}+Z_{i}(x)\right.$ would have also been a valid choice) it is not always possible to construct a continuous map $x \mapsto \partial Z_{i}(x)$. Nonetheless, we argue that a choice of $Z_{1}$ for a given $x$ induces choices of $Z_{2}, \ldots, Z_{N}$ so that if $\tilde{Z}_{i}$ denotes the image of $Z_{i}$ in $\mathcal{C}_{i}$ under the respective bilipschitz diffeomorphism, then $\partial \tilde{Z}_{1}+\ldots+\partial \tilde{Z}_{N}$, as a relative cycle of $M$, does not depend on the choice of $Z_{1}$. Then we show that the map $x \mapsto\left(\partial \tilde{Z}_{1}+\ldots+\partial \tilde{Z}_{N}\right)(x)$ is a $p$-sweepout of $M$ whose elements have masses comparable with those of $\Phi$.

For each $i=1, \ldots, N$ set

$$
S X_{i}=\left\{(x, Z): x \in X, \Phi_{i}(x)-\partial Z \in \mathbf{I}_{n}\left(\partial C_{i} ; \mathbb{Z}_{2}\right)\right\} \subset X \times \mathbf{I}_{n+1}\left(C_{i} ; \mathbb{Z}_{2}\right) .
$$

It is straightforward to see that $S X_{i}$ does not depend on the choice of the representative for $\Phi_{i}(x)$ in $\mathcal{Z}_{n, \text { rel }}\left(C_{i}, \partial C_{i} ; \mathbb{Z}_{2}\right)$. There is a natural projection $\tau_{i}: S X_{i} \rightarrow X, i=1, \ldots, N$.

4.3. Lemma. $\tau_{i}$ is a 2 -cover of $X$ for all $i=1, \ldots, N$.

Proof. Fix $i=1, \ldots, N$.

For every $x \in X$ we have $\Phi_{i}(x)$ in the connected component of zero and so from Proposition 1.23 of [1] one can find $Z_{x} \in \mathbf{I}_{n+1}\left(C_{i} ; \mathbb{Z}_{2}\right)$ so that $\Phi_{i}(x)-\partial Z_{x} \in \mathbf{I}_{n}\left(\partial C_{i} ; \mathbb{Z}_{2}\right)$. Note that $\left(x, C_{i}+Z_{x}\right)$ belongs to $S X_{i}$ as well.

Given $\left(x, Z^{\prime}\right) \in S X_{i}$, then $\partial\left(Z^{\prime}-Z_{x}\right) \in \mathbf{I}_{n}\left(\partial C_{i} ; \mathbb{Z}_{2}\right)$ and so we obtain from the Constancy Theorem [21, page 141] that $Z^{\prime}=Z_{x}$ or $Z^{\prime}=C_{i}+Z_{x}$. As a result, $\tau_{i}^{-1}(x)=\left\{\left(x, Z_{x}\right),\left(x, C_{i}+Z_{x}\right)\right\}$.

The unique lifting property holds for $\pi_{i}$ because $\mathcal{F}\left(Z_{x}, C_{i}+Z_{x}\right)=\left|C_{i}\right|$ for all $x \in X$ and so for $y$ near $x$ there is a unique $Z_{y}$ that is close to $Z_{x}$ in the flat topology.

The isomorphism classes of double covers of $X$ are in a bijective correspondence with $\operatorname{Hom}\left(\pi_{1}(X), \mathbb{Z}_{2}\right)$, which is homeomorphic to $H^{1}\left(X ; \mathbb{Z}_{2}\right)$. We claim that, for all $i=1, \ldots, N$, the element $\sigma_{i} \in H^{1}\left(X ; \mathbb{Z}_{2}\right)$ that classifies $S X_{i}$ is identical to $\lambda$. Indeed given $\gamma: S^{1} \rightarrow X$ nontrivial in $\pi_{1}(X)$, consider a lift to $S X_{i}$ given by $\theta \mapsto\left(\gamma(\exp (i \theta)), Z_{\theta}\right), 0 \leq \theta \leq 2 \pi$. Then $\sigma_{i}(\gamma)$ is 1 if $Z_{0}=C_{i}-Z_{2 \pi}$ and 0 if $Z_{0}=Z_{2 \pi}$. Thus $\sigma_{i}(\gamma)$ is non-zero if and only if $\Phi_{i} \circ \gamma$ is a sweepout. 
As a result we obtain that $S X_{1}$ is isomorphic to $S X_{i}$ for all $i=1, \ldots, N$ and let $F_{i}: S X_{1} \rightarrow S X_{i}$ be the corresponding isomorphism.

For each $i=1, \ldots, N$, there is a natural projection of $S X_{i}$ into $\mathbf{I}_{n+1}\left(C_{i} ; \mathbb{Z}_{2}\right)$ that is continuous in the flat topology. Furthermore $C_{i}$ is bilipschitz diffeomorphic to $\mathcal{C}_{i}$ and so, composing the projection map with that diffeomorphism we obtain $\Xi_{i}: S X_{i} \rightarrow \mathbf{I}_{n+1}\left(\mathcal{C}_{i} ; \mathbb{Z}_{2}\right)$ continuous in the flat topology.

Set

$$
\hat{\Psi}: S X_{1} \rightarrow \mathcal{Z}_{n, \text { rel }}\left(M, \partial M ; \mathbb{Z}_{2}\right), \quad \hat{\Psi}(y)=\sum_{i=1}^{N} \partial\left(\Xi_{i} \circ F_{i}(y)\right) .
$$

The map is continuous in the flat topology.

If $(x, Z) \in S X_{1}$, then $\Xi_{i} \circ F_{i}\left(x, C_{1}+Z\right)=\mathcal{C}_{i}+\Xi_{i} \circ F_{i}(x, Z)$ for all $i=1, \ldots, N$, and so

$$
\begin{aligned}
& \hat{\Psi}\left(x, C_{1}+Z\right)=\sum_{i=1}^{N} \partial\left(\mathcal{C}_{i}+\Xi_{i} \circ F_{i}(x, Z)\right)=\sum_{i=1}^{N} \partial \mathcal{C}_{i}+\hat{\Psi}(x, Z) \\
&=\partial M+\hat{\Psi}(x, Z) .
\end{aligned}
$$

Thus $\hat{\Psi}\left(x, C_{1}+Z\right)=\hat{\Psi}(x, Z)$ in $\mathcal{Z}_{n, \text { rel }}\left(M, \partial M ; \mathbb{Z}_{2}\right)$, which means that $\hat{\Psi}$ descends to a continuous map in the flat topology $\Psi: X \rightarrow \mathcal{Z}_{n, \text { rel }}\left(M, \partial M ; \mathbb{Z}_{2}\right)$.

4.4. Lemma. For all $x \in X$ we have

$$
\mathbf{M}(\Psi(x)) \leq(1+\varepsilon)^{2 n} \mathbf{M}(\Phi(x))+(1+\varepsilon)^{n} \sum_{i=1}^{N}\left|\partial C_{i}\right| .
$$

Proof. Choose $(x, Z) \in S X_{1}$. Then for all $i=1, \ldots, N$, we have that $F_{i}(x, Z)=\left(x, Z_{i}\right) \in S X_{i}$ for some $Z_{i} \in \mathbf{I}_{n+1}\left(C_{i} ; \mathbb{Z}_{2}\right)$ and so we deduce from $\partial Z_{i}-\Phi_{i}(x) \in \mathbf{I}_{n}\left(\partial C_{i}, \mathbb{Z}_{2}\right)$ and (14) that

$$
\mathbf{M}\left(\partial Z_{i}\right) \leq \mathbf{M}\left(\Phi_{i}(x)\right)+\left|\partial C_{i}\right| \leq(1+\varepsilon)^{n} \mathbf{M}\left(\Phi(x)\left\llcorner C_{i}\right)+\left|\partial C_{i}\right| .\right.
$$

Therefore

$$
\sum_{i=1}^{N} \mathbf{M}\left(\partial Z_{i}\right) \leq(1+\varepsilon)^{n} \mathbf{M}(\Phi(x))+\sum_{i=1}^{N}\left|\partial C_{i}\right|
$$

and the result follows because $\mathbf{M}(\Psi(x)) \leq(1+\varepsilon)^{n} \sum_{i=1}^{N} \mathbf{M}\left(\partial Z_{i}\right)$.

Claim : $\Psi$ is a p-sweepout and $\Psi$ has no concentration of mass.

Proof. Choose $\gamma: S^{1} \rightarrow X$ nontrivial in $\pi_{1}(X)$ and denote by $\gamma_{1}$ its lift to $S X_{1}$. Then $\gamma_{i}=F_{i} \circ \gamma_{1}$ gives a lift to $S X_{i}$ for all $i=1, \ldots, N$ and we consider the continuous map in the flat topology

$$
B:[0,2 \pi] \rightarrow \mathbf{I}_{n+1}\left(M ; \mathbb{Z}_{2}\right), \quad B(\theta)=\sum_{i=1}^{N} \Xi_{i} \circ \gamma_{i}(\theta) .
$$


We have $(\Psi \circ \gamma)(\theta)=\partial B(\theta)$ for all $0 \leq \theta \leq 2 \pi$.

Hence $\Psi^{*} \bar{\lambda}=\lambda$ because, recalling that $\sigma_{i}=\lambda$ for all $i=1, \ldots, N$,

$$
\begin{aligned}
\lambda(\gamma)=0 & \Longrightarrow \sigma_{i}(\gamma)=0 \text { for all } i=1, \ldots, N \\
& \Longrightarrow \Xi_{i} \circ \gamma_{i}(2 \pi)=\Xi_{i} \circ \gamma_{i}(0) \text { for all } i=1, \ldots, N \\
& \Longrightarrow B(2 \pi)=B(0)
\end{aligned}
$$

and

$$
\begin{aligned}
\lambda(\gamma)=1 & \Longrightarrow \sigma_{i}(\gamma)=1 \text { for all } i=1, \ldots, N \\
& \Longrightarrow \Xi_{i} \circ \gamma_{i}(2 \pi)=\mathcal{C}_{i}+\Xi_{i} \circ \gamma_{i}(0) \text { for all } i=1, \ldots, N \\
& \Longrightarrow B(2 \pi)=M+B(0),
\end{aligned}
$$

where in the last line we used the fact that $\left\{\mathcal{C}_{i}\right\}_{i=1}^{N}$ are pairwise disjoint and cover $M$.

This implies that $\Psi$ is a $p$-sweepout because $\lambda^{p} \neq 0$. We leave to the reader to check that $\Psi$ has no concentration of mass.

From Corollary 2.13, Lemma 4.4, and the previous claim we obtain

$$
\omega_{p}(M) \leq(1+\varepsilon)^{2 n} \omega_{p}(\Omega)+(1+\varepsilon)^{n} \sum_{i=1}^{N}\left|\partial C_{i}\right| .
$$

Dividing the inequality above by $p^{1 /(n+1)}$, making $p \rightarrow \infty$, and using Theorem 3.2 we have

$$
\limsup _{p \rightarrow \infty} \tilde{\omega}_{p}(M) \leq a(n)(1+\varepsilon)^{2 n}|\Omega|^{n /(n+1)} .
$$

Using (13) and making $\varepsilon$ tend to zero in the two inequalities we obtain

$$
\limsup _{p \rightarrow \infty} \tilde{\omega}_{p}(M) \leq a(n)(\operatorname{vol} M)^{n /(n+1)} .
$$

This inequality and Theorem 4.1 imply the desired result.

\section{Appendix A}

Proof of Theorem 2.10. Set $a(q)=2^{-4(q+2)^{2}-2}$ where $q \in \mathbb{N}$ is fixed. We use $\mathbf{B}_{r}^{\mathcal{F}}(T)$ to denote the ball of radius $r$ in the flat topology centred at $T \in \mathcal{Z}_{k, \text { rel }}\left(M, \partial M ; \mathbb{Z}_{2}\right)$. Finally $I_{0}(m, l)$ denotes the cells of $I(m, l)$ whose support lie in $\partial I^{m}$.

The key step consists in proving the following lemma below:

Given $T \in \mathcal{Z}_{k, \text { rel }}\left(M, \partial M ; \mathbb{Z}_{2}\right)$ with $\mathbf{M}(T) \leq L, l \in \mathbb{N}$, and $m \leq q+1$, assume there is a sequence

$$
\phi_{k}: I_{0}(m, l)_{0} \rightarrow \mathbf{B}_{\varepsilon_{k}}^{\mathcal{F}}(T) \cap\{S: \mathbf{M}(S) \leq 2 L\}
$$

with $\varepsilon_{k}<1 / k$ and $\mathbf{m}\left(\phi_{k}, r\right) \leq \delta / 4$. 
A.1. Lemma. There exists $N \in \mathbb{N}, N \geq l$, such that for a subsequence $\left\{\phi_{j}\right\}$ of $\left\{\phi_{k}\right\}_{k \in \mathbb{N}}$ we can find

$$
\psi_{j}: I(1, N)_{0} \times I_{0}(m, l)_{0} \rightarrow \mathbf{B}_{\varepsilon_{j}}^{\mathcal{F}}(T)
$$

satisfying

(i) $\mathbf{f}\left(\psi_{j}\right) \leq \delta$ if $m=1$ and $\mathbf{f}\left(\psi_{j}\right) \leq \mathbf{f}\left(\phi_{j}\right)+\delta$ if $m \neq 1$;

(ii) $\psi_{j}([0], x)=\phi_{j}(x)$ and $\psi_{j}([1], x)=T$ for all $x \in I_{0}(m, l)_{0}$;

(iii)

$$
\begin{aligned}
\sup \left\{\mathbf{M}\left(\psi_{j}(y, x)\right):(y, x) \in I(1, N)_{0} \times\right. & \left.I_{0}(m, l)_{0}\right\} \\
& \leq \sup _{x \in I_{0}(m, l)_{0}}\left\{\mathbf{M}\left(\phi_{j}(x)\right)\right\}+\frac{\delta}{n+1} ;
\end{aligned}
$$

(iv) $\mathbf{m}\left(\psi_{j}, r\right) \leq 2\left(\mathbf{m}\left(\phi_{j}, r\right)+a(n) \delta\right)$.

Once this result is proven, Theorem 2.10 follows exactly in the same way that Theorem 13.1 in [15] followed from [15, Lemma 13.4].

Proof. From Proposition 2.4 we can assume that $\left\|\phi_{k}(x)\right\|(\partial M) \leq 1 / k$ for all $k \in \mathbb{N}$ and $x \in I_{0}(m, l)_{0}$. Since the set of varifolds in $\mathcal{V}_{k}(M)$ with mass bounded above by $2 L$ is compact in the weak topology, we can find a subsequence $\left\{\phi_{j}\right\}$ of $\left\{\phi_{k}\right\}_{k \in \mathbb{N}}$ and a map

$$
V: I_{0}(m, l)_{0} \rightarrow \mathcal{V}_{k}(M)
$$

so that

$$
\lim _{j \rightarrow \infty}\left|\phi_{j}(x)\right|=V(x) \text { as varifolds, }
$$

for each $x \in I_{0}(m, l)_{0}$.

Note that $\mathcal{F}\left(\phi_{j}(x), T\right)$ tends to 0 as $j \rightarrow \infty$ (as relative cycles). Thus from Proposition 2.4 and since $\mathbf{m}\left(\phi_{j}, r\right) \leq \delta / 4$, we have

$$
\|T\|\left(B_{r}(p) \backslash \partial M\right) \leq \mathbf{m}\left(\phi_{j}, r\right)+a(n) \delta<\frac{\delta}{3}
$$

and

$$
\|V\|\left(B_{r}(p)\right) \leq \mathbf{m}\left(\phi_{j}, r\right)+a(n) \delta<\frac{\delta}{3}
$$

for all $j$ sufficiently large, $p \in M$, and $x \in I_{0}(m, l)_{0}$.

We can choose points $\left\{p_{i}\right\}_{i=1}^{v}$, and positive real numbers $\left\{r_{i}\right\}_{i=1}^{v}, r_{i}<r$, so that

$$
B_{r_{i_{1}}}\left(p_{i_{1}}\right) \cap B_{r_{i_{2}}}\left(p_{i_{2}}\right)=\emptyset \quad \text { if } i_{1} \neq i_{2}
$$

and such that

$$
\begin{gathered}
\|T\|\left(\partial B_{r_{i}}\left(p_{i}\right)\right) \leq\|V(x)\|\left(\partial B_{r_{i}}\left(p_{i}\right)\right)=0, \\
\|V(x)\|\left(M \backslash \cup_{i=1}^{v} B_{r_{i}}\left(p_{i}\right)\right)<\frac{\delta}{3},
\end{gathered}
$$


and

$$
\|T\|\left(B_{r_{i}}\left(p_{i}\right) \backslash M\right) \leq\|V(x)\|\left(B_{r_{i}}\left(p_{i}\right)\right)=\lim _{j \rightarrow \infty}\left\|\phi_{j}(x)\right\|\left(B_{r_{i}}\left(p_{i}\right)\right)<\frac{\delta}{3},
$$

for all $x \in I_{0}(m, l)_{0}$ and $i=1, \ldots, v$. We can assume $v=3^{N}-1$ for some $N \in \mathbb{N}$ satisfying $N \geq l$.

From [1, Proposition 1.23], we get that there exists $Q_{j}(x) \in \mathbf{I}_{k}(M)$, $R_{j}(x) \in \mathbf{I}_{k}(\partial M)$ for all $j$ sufficiently large and $x \in I_{0}(m, l)_{0}$, such that

$$
\partial Q_{j}(x)=\phi_{j}(x)-T+R_{j}(x), \quad \mathbf{M}\left(Q_{j}(x)\right)=\mathcal{F}\left(\phi_{j}(x)-T\right) .
$$

In particular we have $\mathbf{M}\left(Q_{j}(x)\right)<\varepsilon_{j}<1 / j$.

For each $i=1, \ldots, v$, consider the distance function $d_{i}(x)=d\left(p_{i}, x\right)$. Using [21, Lemma 28.5], we find a decreasing subsequence $\left\{r_{i}^{j}\right\}$ converging to $r_{i}$ with $r_{i}^{j}<r$ and such that the slices $\left\langle Q_{j}(x), d_{i}, r_{i}^{j}\right\rangle$ are in $\mathbf{I}_{k}(M)$ and satisfy

$$
\begin{gathered}
\left\langle Q_{j}(x), d_{i}, r_{i}^{j}\right\rangle=\partial\left(Q_{j}(x)\left\llcorner B_{r_{i}^{j}}\left(p_{i}\right)\right)-\left(\phi_{j}(x)-T+R_{j}(x)\right)\left\llcorner B_{r_{i}^{j}}\left(p_{i}\right),\right.\right. \\
\phi_{j}(x)\left\llcorner B_{r_{i}^{j}}\left(p_{i}\right), T\left\llcornerB _ { r _ { i } ^ { j } } ( p _ { i } ) \in \mathbf { I } _ { k } ( M ) , \quad \text { and } \quad R _ { j } ( x ) \left\llcorner B_{r_{i}^{j}}\left(p_{i}\right) \in \mathbf{I}_{k}(\partial M)\right.\right.\right.
\end{gathered}
$$

for every $x \in I_{0}(m, l)_{0}$. Note that since $\lim _{j \rightarrow \infty} \mathbf{M}\left(Q_{j}(x)\right)=0$, by the coarea formula we can choose $\left\{r_{i}^{j}\right\}$ such that

$$
\sum_{x \in I_{0}(m, l)_{0}} \sum_{i=1}^{v} \mathbf{M}\left(\left\langle Q_{j}(x), d_{i}, r_{i}^{j}\right\rangle\right) \leq a(n) \delta<\frac{\delta}{2(n+1)}
$$

for every sufficiently large $j$. Furthermore, using (16), (17), (18), and Proposition 2.4 , we get that

$$
\begin{gathered}
\left\|\phi_{j}(x)\right\|\left(B_{r_{i}^{j}}\left(p_{i}\right)\right)<\frac{\delta}{3}, \quad\|T\|\left(B_{r_{i}^{j}}\left(p_{i}\right) \backslash M\right)<\frac{\delta}{3}, \\
\left\|\phi_{j}(x)\right\|\left(M \backslash \cup_{i=1}^{v} B_{r_{i}}\left(p_{i}\right)\right)<\frac{\delta}{3}, \quad\|T\|\left(M \backslash\left(\cup_{i=1}^{v} B_{r_{i}}\left(p_{i}\right) \cup \partial M\right)\right)<\frac{\delta}{3},
\end{gathered}
$$

and

$$
\left(\|T\|-\left\|\phi_{j}(x)\right\|\right)\left(B_{r_{i}^{j}}\left(p_{i}\right) \backslash \partial M\right) \leq \frac{\delta}{2(n+1) v}
$$

for every sufficiently large $j, i=1, \ldots, v$, and $x \in I_{0}(m, l)_{0}$.

We consider the map given by

$$
\begin{aligned}
\psi_{j}\left(\left[\frac{i}{3^{N}}\right], x\right) & =\phi_{j}(x)-\sum_{a=1}^{i} \partial\left(Q_{j}(x)\left\llcorner B_{r_{a}^{j}}\left(p_{a}\right)\right) \quad \text { if } 0 \leq i \leq 3^{N}-1,\right. \\
\psi_{j}([1], x) & =T
\end{aligned}
$$

defined on $I(1, N)_{0} \times I_{0}(m, l)_{0}$.

Note that as relative cycles

$$
\psi_{j}\left(\left[\frac{i}{3^{N}}\right], x\right)-T=\partial\left(Q _ { j } ( x ) \left\llcorner\left(M \backslash \cup_{a=1}^{i} B_{r_{a}^{j}}\left(p_{a}\right)\right),\right.\right.
$$


from which it follows that $\psi_{j}\left(\left[\frac{i}{3^{N}}\right], x\right) \in \mathbf{B}_{\varepsilon_{j}}^{\mathcal{F}}(T)$. From (19), we also have that as relative cycles

$$
\begin{aligned}
\psi_{j}\left(\left[\frac{i}{3^{N}}\right], x\right)=\phi_{j}(x)\left\llcorner\left(M \backslash \cup_{a=1}^{i} B_{r_{a}^{j}}\left(p_{a}\right)\right)+\sum_{a=1}^{i} T\left\llcorner B_{r_{a}^{j}}\left(p_{a}\right)\right.\right. \\
-\sum_{a=1}^{i}\left\langle Q_{j}(x), d_{a}, r_{a}^{j}\right\rangle\left\llcorner B_{r_{a}^{j}}\left(p_{a}\right) .\right.
\end{aligned}
$$

In what follows the masses of currents are always computed as relative cycles, i..e, using Proposition 2.4. From (20), (21), (22), and (24) we have that

$$
\begin{array}{r}
\mathbf{M}\left(\psi_{j}\left(\left[\frac{i}{3^{N}}\right], x\right)-\psi_{j}\left(\left[\frac{i-1}{3^{N}}\right], x\right)\right) \\
\leq \frac{\delta}{3}+\left\|\phi_{j}(x)\right\|\left(B_{r_{i}^{j}}\left(p_{i}\right)\right)+\|T\|\left(B_{r_{i}^{j}}\left(p_{i}\right) \backslash M\right)<\delta
\end{array}
$$

for $1 \leq i \leq v=3^{N}-1$, and

$$
\begin{aligned}
\mathbf{M}\left(\psi_{j}\left(\left[1-\frac{1}{3^{N}}\right], x\right)-T\right) & \leq\left\|\phi_{j}(x)\right\|\left(M \backslash \cup_{a=1}^{v} B_{r_{a}^{j}}\left(p_{a}\right)\right) \\
& +\|T\|\left(M \backslash\left(\cup_{a=1}^{v} B_{r_{a}^{j}}\left(p_{a}\right) \cup \partial M\right)\right)+\frac{\delta}{3}<\delta .
\end{aligned}
$$

If $\mathbf{d}(x, y)=1$, we also have

$$
\begin{aligned}
\mathbf{M}\left(\psi_{j}\left(\left[\frac{i}{3^{N}}\right], x\right)-\psi_{j}\left(\left[\frac{i}{3^{N}}\right], y\right)\right) \\
\leq\left\|\phi_{j}(x)-\phi_{j}(y)\right\|(M \backslash M)+\frac{\delta}{2} \\
\quad \leq \mathbf{f}\left(\phi_{j}\right)+\delta .
\end{aligned}
$$

Hence $\mathbf{f}\left(\psi_{j}\right) \leq \mathbf{f}\left(\phi_{j}\right)+\delta$.

To prove Lemma A.1(iii) we use (20), (23), and (24), to conclude

$$
\begin{aligned}
\mathbf{M}\left(\psi_{j}\left(\left[\frac{i}{3^{N}}\right], x\right)\right) \leq & \left\|\phi_{j}(x)\right\|\left(M \backslash\left(\cup_{a=1}^{i} B_{r_{a}^{j}}\left(p_{a}\right) \cup \partial M\right)\right) \\
& \quad+\sum_{a=1}^{i}\|T\|\left(B_{r_{a}^{j}}\left(p_{a}\right) \backslash \partial M\right)+\frac{\delta}{2(n+1)} \\
\leq & \left\|\phi_{j}(x)\right\|(M \backslash M) \\
& \quad+\sum_{a=1}^{i}\left(\|T\|-\left\|\phi_{j}(x)\right\|\right)\left(B_{r_{a}^{j}}\left(p_{a}\right) \backslash M\right)+\frac{\delta}{2(n+1)} \\
\leq & \left\|\phi_{j}(x)\right\|(M \backslash M)+\frac{\delta}{n+1}=\mathbf{M}\left(\phi_{j}(x)\right)+\frac{\delta}{n+1} .
\end{aligned}
$$


Finally, Lemma A.1(iv) follows from (15), (20), and (24):

$$
\begin{aligned}
\left\|\psi_{j}\left(\left[\frac{i}{3^{N}}\right], x\right)\right\|\left(B_{r}(p) \backslash \partial M\right) & \leq \\
\left\|\phi_{j}(x)\right\|\left(B_{r}(p) \backslash \partial M\right)+\|T\|\left(B_{r}(p) \backslash \partial M\right)+a(n) \delta & \leq 2 \mathbf{m}\left(\phi_{k}, r\right)+2 a(n) \delta .
\end{aligned}
$$

\section{ApPendix B}

Proof of Theorem 2.11. The analogous result for continuous functions in the flat topology was proven by Almgren in Theorem 6.6 [1]. For continuous functions in the mass topology this result was proven in Theorem 14.1 in [15] when $k=n$ and $\partial M=\emptyset$ by adapting the proof of [1, Theorem 6.6]. We now explain which further adaptations need to be made in order to prove Theorem 2.11.

The constant $\delta_{0}$ is chosen so that Theorem 2.5 in [1] can be applied and thus we obtain a chain map (defined in [1, Definition 2.3]) $\phi_{M}: Y \rightarrow$ $\mathbf{I}_{*}\left(M ; \mathbb{Z}_{2}\right)$ of degree $k$ so that $\phi_{M}=\phi$ on $Y_{0}$ and

$$
\mathbf{M}\left(\phi_{M}(\alpha)\right) \leq 2 \mathbf{f}(\phi) \text { for all } \alpha \in Y_{p}, p \geq 1 .
$$

Consider a differentiable triangulation of $M$, the deformation map $\mathcal{D}$ given in [20, Theorem 4.5] that is continuous in the mass topology, and the cutting function given by [1, Theorem 5.8]. For every $\alpha \in Y_{p}, p \geq 1$, one has now all the necessary ingredients to consider the function

$$
h_{\alpha}: \alpha \rightarrow \mathcal{Z}_{k}\left(M, \partial M ; \mathbb{Z}_{2}\right)
$$

given by [1, Interpolation Formula 6.3] (with $A=M, B=\partial M, \phi_{A}=\phi_{M}$, and $\left.\phi_{B}=0\right)$. The projection

$$
\tilde{h}_{\alpha}: \alpha \rightarrow \mathcal{Z}_{k, \operatorname{rel}}\left(M, \partial M ; \mathbf{M} ; \mathbb{Z}_{2}\right)
$$

is continuous in the mass topology (see [1, page 297]).

Using the maps $\left\{\tilde{h}_{\alpha}\right\}_{\alpha \in Y}$ and the construction described in [1, Section 6.5] one obtains the map $\Phi$ satisfying (i) and (ii). Property (iii) also follows because Theorem 6.62 (b) of [1] (see also [15, Lemma 14.4]) translates into the fact that if $x, y$ lie in a common cell of $Y$ then for some $C=C(M, m)$ we have

$$
\mathbf{M}(\Phi(x)-\Phi(y)) \leq C \sup \left\{\mathbf{M}\left(\phi_{M}(\alpha)\right): \alpha \in Y_{p}, p \geq 1\right\} \leq 2 C \mathbf{f}(\phi) .
$$




\section{REFERENCES}

[1] F. Almgren, The homotopy groups of the integral cycle groups, Topology (1962), 257299.

[2] H. Federer, Geometric measure theory, Die Grundlehren der mathematischen Wissenschaften, Band 153 Springer-Verlag New York Inc., New York 1969.

[3] H. Federer and W. Fleming, Normal and integral currents, Ann. of Math. 72 (1960) 458-520.

[4] P. Glynn-Adey and Y. Liokumovich, Width, Ricci curvature and minimal hypersurfaces, J. Differential Geom. 105 (2017), no. 1, 33-54.

[5] M. Gromov, Dimension, nonlinear spectra and width,. Geometric aspects of functional analysis,(1986/87), 132-184, Lecture Notes in Math., 1317, Springer, Berlin, 1988.

[6] M. Gromov, Isoperimetry of waists and concentration of maps, Geom. Funct. Anal. 13 (2003), 178-215.

[7] M. Gromov, Singularities, expanders and topology of maps. I. Homology versus volume in the spaces of cycles. Geom. Funct. Anal. 19 (2009), 743-841.

[8] M. Gromov, Morse spectra, homology measures, spaces of cycles and parametric packing problem, preprint, 2015.

[9] L. Guth, Minimax problems related to cup powers and Steenrod squares, Geom. Funct. Anal. 18 (2009), 1917-1987.

[10] A. Hatcher, Algebraic Topology, Cambridge University Press (2002)

[11] S. Hofmann, M. Mitrea, and M. Taylor, Geometric and transformational properties of Lipschitz domains, Semmes-Kenig-Toro domains, and other classes of finite perimeter domains, J. Geom. Anal. 17 (2007), 593-647.

[12] K. Irie, F. C. Marques and A. Neves, Denseness of minimal hypersurfaces for generic metrics, arXiv:1710.10752 [math.DG] (2017)

[13] N. Korevaar, Upper bounds for eigenvalues of conformal metrics. J. Different. Geom. 37, 1, 73-93, 1993.

[14] Y. Liokumovich, Families of short cycles on Riemannian surfaces, Duke Math. J. 165 (2016), no. 7, 136-1379.

[15] Marques, F. C., Neves A., Min-max theory and the Willmore conjecture, Ann. of Math. 1792 (2014), 683-782.

[16] Marques, F. C., Neves, A., Existence of infinitely many minimal hypersurfaces in positive Ricci curvature, Invent. Math. 209 (2017), no.2, 577-616.

[17] Marques, F. C., Neves, A., Applications of Almgren-Pitts min-max theory, Current developments in mathematics 2013, 1-71, Int. Press, Somerville, MA, 2014.

[18] Marques, F. C., Neves, A., Topology of the space of cycles and existence of minimal varieties, Surveys in Differential Geometry, 21 (2016), 165-177, International Press.

[19] P. Papasoglu, E. Swenson, A sphere hard to cut, arXiv:1509.02307 [math.DG].

[20] J. Pitts, Existence and regularity of minimal surfaces on Riemannian manifolds, Mathematical Notes 27, Princeton University Press, Princeton, (1981).

[21] L. Simon, Lectures on geometric measure theory, Proceedings of the Centre for Mathematical Analysis, Australian National University, Canberra, (1983).

[22] H. Weyl, Über die Asymptotische Verteilung der Eigenwerte, Nachr. Konigl. Ges. Wiss. Göttingen (1911), 110-117.

[23] S.-T. Yau, Problem section. Seminar on Differential Geometry, pp. 669-706, Ann. of Math. Stud., 102, Princeton Univ. Press, Princeton, N.J., 1982. 
Department of Mathematics, Massachusetts Institute of Technology, CamBRIDGE, MA 02139

Princeton University, Fine Hall, Princeton, NJ 08544

University of Chicago, Department of Mathematics, Chicago IL 60637, USA /Imperial College London, Huxley Building, 180 Queen's Gate, London SW7 2RH, United Kingdom

E-mail address: ylio@mit.edu

E-mail address: coda@math.princeton.edu

E-mail address: aneves@uchicago.edu, a.neves@imperial.ac.uk 